HORST BEINLICH

\title{
Zwei Osirishymnen in Dendera
}

$\mathrm{Zu}$ den längsten Texten in den Osirisräumen auf dem Dach des Tempels von Dendera gehören mit insgesamt 80 Zeilen die beiden Hälften eines Hymnus, der Osiris in allen Gauen $\ddot{A}$ gyptens preist ${ }^{1}$. An den beiden Seiten des 1. Raumes, also des Lichthofes, der westlichen Osirisräume $^{2}$ angebracht, nimmt er eine Position ein, die weitgehend der des großen Choiaktextes $^{3}$ im Lichthof der östlichen Osirisräume entspricht. Zusammen mit einem sehr viel kürzeren Paralleltext ${ }^{4}$ am Eingang des Raumes $\mathrm{F}$ in Dendera ${ }^{5}$ hat der Hymnus schon früh die Aufmerksamkeit der Ägyptologie auf sich gelenkt, weil hier Osiriskultorte in ganz Ägypten genannt sind. Unabhängig voneinander legten zwei Gelehrte fast gleichzeitig ihre Textabschriften vor, zunächst Johannes Dümichen 1865 im ersten Band seiner „Geographischen Inschriften“6 , dann 1873 Auguste Mariette im 4. Band seiner Publikation des Dendera-Tempels ${ }^{7}$. Die Informationen über lokale Gegebenheiten des Osiriskultes, vor allem aber über die Toponyme innerhalb der Inschrift sind über die beiden großen Nachschlagewerke der ägyptischen Geographie von Heinrich Brugsch und Henri Gauthier ${ }^{8}$ schnell Allgemeingut geworden. Wohl keine Arbeit, die sich heute mit ägyptischen Gauen beschäftigt, kann ohne diese Quellen auskommen. So ist es höchst verwunderlich, daß zu der Hymne bisher keine zusammenhängende Übersetzung und Bearbeitung existiert, wenngleich auch viele einzelne Passagen auszugsweise übersetzt sind ${ }^{9}$. Aus diesem Grund ist auch die kunstvolle Gliederung des Textes und sein Wert über die topographische Bedeutung hinaus unbeachtet geblieben.

Es ist sicher leicht einzusehen, daß auf den wenigen Seiten eines Aufsatzes eine umfassende Kommentierung des gesamten Textes nicht möglich ist, da sie z. B. auf die Problematik der Lokalisierung aller Ortsnamen eingehen müßte. Ich beschränke deshalb im ,geographischen Teil“ den Kommentar auf die Angabe von Textparallelen aus dem Bereich der „Geographischen Inschriften“. Eine Beschränkung auf diese Textgruppe erscheint mir deshalb sinnvoll, weil so die gemeinschaftliche Verwendung von Wort- und Sinnzusammenhängen durch die Priesterschaften unterschiedlicher Tempel deutlicher wird, bei der nicht das Abschreiben der gleichen Quelle die schriftlichen Formulierungen der Endfassung bestimmt, sondern das gemeinsame, mündliche oder schriftliche Hintergrundwissen dem Textredakteur in diesem Rahmen die Freiheit einer eigenen schöpferischen Leistung läßt.

\footnotetext{
${ }^{1} \mathrm{Im}$ folgenden wird dieser Text als „Text A“ bezeichnet.

${ }^{2}$ P.-M. VI, S. 94 (12-13 u. 16-17), s. a. Skizze S. 25.

${ }^{3}$ Chassinat, Le mystère d'Osiris au mois de Khoiak, 1966-1968.

${ }^{4} \mathrm{Im}$ folgenden wird dieser Text als „Text B“ bezeichnet.

${ }^{5}$ P.-M. VI, S. 52 (163a-b).

${ }^{6}$ S. u. S. 31.

7 S. u. S. 31.

${ }^{8}$ Brugsch, DG; Gauthier, DG.

${ }^{9}$ Es ist unmöglich, hier alle Übersetzungen oder gar Erwähnungen zu zitieren. Man muß damit rechnen, daß in jeder Arbeit, die sich mit Ortsnamen im alten Ägypten beschäftigt, sei es in Arbeiten über ganz Ägypten, sei es in solchen über einzelne Gaue, eine Vielzahl von Zitaten aus Text A und Text B zu finden ist.
} 


\section{Text A (M. D. IV, pl. 73 u. 75)}

Publikation: Auguste Mariette: Dendérah, Tome IV, Planches, Paris 1873, pl. 73 u. 75. Johannes Dümichen: Geographische Inschriften altägyptischer Denkmäler, Erste Abtheilung - Tafeln (= Brugsch: Recueil de monuments égyptiens, Bd. III), Leipzig 1865, Taf. 96-100 ${ }^{10}$.

Die beiden Publikationen des Textes sind relativ genau, was die Abfolge der Hieroglyphen betrifft. Da es sich um zwei unabhängige Abschriften handelt, lassen sich Fehler mit einiger Wahrscheinlichkeit erkennen. Wenn man sie einzeln werten und vergleichen sollte, müßte man sagen, daß die Wiedergabe von Mariette dem Original besser entspricht. So sind die beiden Darstellungen, die vom Text umfaßt werden, von Mariette in Bild und Beischrift zufriedenstellend abgebildet. Dümichen läßt die Beischrift auf der einen Seite fast völlig weg, und die Wiedergabe der dargestellten Figuren hat mit der Realität nicht viel gemein. Unzuverlässig ist bei Dümichen auch der Zeilenumbruch. Andererseits findet man nur bei Dümichen die waagerechte Textzeile unterhalb der unterägyptischen Hälfte der Hymne ${ }^{11}$, das Pendant auf der gegenüberliegenden Wand ist leider völlig unpubliziert. Man hat den Eindruck, daß Dümichen den Text in großer Eile abgeschrieben hat, denn es sind ihm einige Flüchtigkeitsfehler unterlaufen, und Abmessungen zerstörter Textstellen sind nicht zuverlässig. Andererseits merkt man seiner Abschrift aber auch einen durch Erfahrung mit Spätzeittexten geschulten Blick an. Wo Mariette machmal nur Zeichenreste wiedergibt, erkennt Dümichen, wohl aus dem Wissen, was dagestanden haben muß, die Hieroglyphe. Beide Textabschriften sind also parallel solange heranzuziehen, bis im Rahmen der Dendera-Publikation eine zuverlässigere Abschrift vorgelegt wird. Bei der unten vorgelegten Übersetzung folge ich zunächst der Version von Mariette und merke Korrekturen, die sich aus der Textversion von Dümichen ergeben, an, wenn sie für die Übersetzung der Textstelle relevant sind.

Die beiden Textausgaben zählen die Zeilen jeweils für eine Texthälfte von 1 bis 40, ohne dabei die Texthälften zu unterscheiden. Im Nachfolgenden ist zur Unterscheidung bei Zeilen der Ostwand, wo die oberägyptische Texthälfte zu finden ist, der Zeilenzahl ein „O“ vorangestellt (z.B. Z.O-28), entsprechend bei der Westwand mit der unterägyptischen Texthälfte ein „W“ (z. B. Z. W-28).

\section{Übersetzung von Text A:}

[ ]= nur in Abschrift Dümichen

Text A (Ostwand)

|1| Osiris anbeten, seinen $\mathrm{Ka}$ in [Oberägypten] preisen. [Sprechen:]

\section{Text A (Westwand)}

|1| Osiris anbeten, seinen $\mathrm{Ba}(?)$ in Unterägypten preisen. Sprechen:

\footnotetext{
${ }^{10}$ Die Dümichen-Abschrift ist die Grundlage der Wörterbuchzettel (1289-1354), die von Text A vorhanden sind. Sie stammen von der Hand Junkers und haben - mit Lücken - eine durchgehende Übersetzung. Gleiches gilt für den Text B (Wörterbuchzettel 1267-1288). Ich möchte an dieser Stelle Herrn Prof. Reineke und Frau Dr. Burkhardt für die freundliche Überlassung von Kopien dieser Zettel danken.

${ }^{11}$ A.a. O., Taf. $98-100$.
} 


\section{(1. o.äg. Gau) ${ }^{12}$}

Bist du in Elephantine ? - so bist du in der „Stadt des Füllens“(13 $|2|$ Es fließt (hwj) für dich der Nil (Hapi) als dein Ausfluß ${ }^{14}$.

Deine Schwester ist die Sothis. [Er] (der Nil) ergießt sich zu seiner Zeit ${ }^{15}$. Chnum ${ }^{16}$ bist du, der alle Dinge erzeugt ${ }^{17}$.

\section{(2. o.äg. Gau $)^{21}$}

|3| Bist du in Behedet (Edfu) ? - so bist du in Mesen (der Harpuniererstätte) der geflügelten Sonnenscheibe. Du betrittst ihr Heiligtum ( ${ }^{3} j j . t$. . Dein Sohn Horus $|4|$ ist auf dem Thron $(m n-b j t)^{22}$, mächtig als Herr des Thronsitzes $\left(\underline{t} \underline{\underline{t}} \underline{\jmath}^{3} . t\right)$.

\section{(3. o.äg. Gau $)^{26}$}

Bist du in Fag $|5|$ als Herr auf dem ...-Tempel ${ }^{27}$ ? - so erstrahlt „die-die-Flügel-ausbreitet ${ }^{\text {‘28 }}$ auf deinem Kopf. Du empfängst den süßen Windhauch von dem, der (oder: von der, die ...) deine Gestalt geschaffen hat ${ }^{29}$. $|6|$ Man reinigt deinen Leib mit Natron ${ }^{30}$.

\section{(1. u.äg. Gau $)^{18}$}

Bist du im Gau „Weiße Mauer“, im Inneren des Goldhauses $^{19}-|2|$ so ist ḩwt-ḩm ${ }^{3} g$ und (?) das Haus der Henu-Barke für deine Mumie $\left(s^{c} \not\right)$ und deinen Ba. Der Setem-Priester macht deine Mundöffnung für deine Knochen. Er gießt aus $(s t \underline{)})$ Wasser $(s t f)$ für deine Statue $(z n n)^{20}$.

\section{(2. u.äg. Gau) ${ }^{23}$}

|3| Bist du [in] Imhedjet ${ }^{24}$, im Gebiet von Chem, dem großen Platz aller Götter Ägyptens (snw.t) ? - so ist ausgestattet die $|4|$ Ausrüstung mit deinen Dingen. Benen ${ }^{25}$ ist der Schutz hinter deiner Gestalt (bs).

\section{(3. u.äg. Gau) ${ }^{31}$}

Bist du in Imaw, der Stadt des $|5|$ Apis ? - so ist deine Macht größer als die der Götter. Alle Leute $(b w-n b)$ libieren Wasser für den Schöpfer (?) ihres Leibes. Der, der deinen Namen nennt $(d m-r n-k)$, ist $|6|$ der Erste ihres Vaters (? ḩ ว.t $j t-s n)(?)$.

\footnotetext{
${ }^{12}$ Text B $=$ CD II, 132,11: „Bist du in Elephantine? - so bist du in der Stadt des Füllens, entstanden an der Spitze ( $m$ ḅ̧.t) aller Götter.“

${ }^{13}$ „Stadt des Füllens“: Tôd, Nr. 82; Philae 88 (IV); „Du bist in der ,Stadt des Füllens` an der Spitze aller Götter“: Opet 210. Durch ,an der Spitze aller Götter“ (vgl. Text B = CDII, 132,11) wird auf den Begriff „Anfang der Gaue" angespielt, wie wir ihn in E. VII, 297f. (297,10; 298,2; 298,4), RdM V, 3 (2×); E. V. 199,1f. finden.
}

${ }^{14}$ E. V, 106,8 und Opet 210: „Hapi quillt hervor als sein Ausfluß.“

${ }^{15}$ CD I, 91: „Du (Hathor) bist Sothis, die den Nun hervorquellen läßt am Jahresanfang“; RdM V, 59: „Du (Hathor) bist das Auge des Re, Sothis, die den Nil hervorquellen läßt zu seiner Zeit". Das „n.f “ unter „sw" ist im Original deutlich zu erkennen.

${ }^{16}$ Nach Dümichen. Mariette ergänzt die fast völlig zerstörte Stelle „Osiris bist du“.

17 Tôd, Nr. 82: „Hapi bist du ( $b^{c} p j$ j-k)“; Philae 88 (IV): „Chnum bist du, der die Befeuchtung erzeugt“ (? ntf - vom Nil, der das Land benetzt: WB II, 356,7); RdM V, 3: „Chnum bist du, der alle Dinge erzeugt“; Opet 210: „Chnum ist es, der alle Dinge erzeugt".

${ }^{18}$ Text B = CD II, 133,8: „Bist du im Gau, Weiße Mauer in $t p h . t-n n$ ? - so erzeugt das Haus des $r s-w \underline{d} s^{3}$ deine

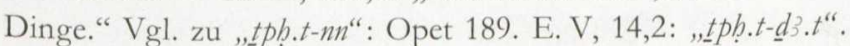

${ }^{19}$ Vgl. dazu: RdM V, 16; RdM III, 82; Osirisreliquien, S. 154.

${ }^{20}$ Opet 189: „Er gießt Wasser für dich aus $(s t j-f n-k, m w) “$.

${ }^{21}$ Text B = CD II, 132,11-133,1: „Bist du in Behedet des Horussees ? - so bin (ich) neben dir als Horus von Behedet.“ Zum „Horussee“ s. Opet 210: „Er kommt aus Djeba des Horussees in Behedet. Der sehr buntgefiederte, er ist neben dir als Horus von Behedet im Inneren des Thrones der Götter.“ Mit dem Namen „Horussee“ verbindet man wohl die Vorstellung von Horus, dem Harpunierer, vgl. Alliot, Culte d'Horus, S. 764 f. u. S. 691 . Osiris ist hier also von einem wehrhaften Gott geschützt.

${ }^{22}$ E. IV, 173,9: „Du bist Horus, der Herr des Lebens, auf dem Thron (mn-bjt)“.

${ }^{23}$ Text B = CD II, 133,8-134,1: „Bist du in Imhedjet, im Gebiet von Chem ? - so bist du erhaben (tnj) als Herrscher des Westens. “

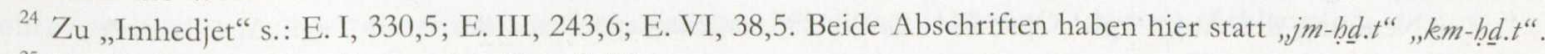

${ }^{25} \mathrm{Zu}$ „Benen“"s. Vandier, pJumilhac S. 75. 
(4. o.äg. Gau $)^{32}$

Bist du in Theben, der Herrin der göttlichen Gaue? - so ist es der Platz deines Herzen, |7| wo du geboren bist ${ }^{33}$. Größer ist deine Macht als alle Götter vereint. Verjüngt ist der König, der die weiße Krone trägt.

\section{(5. o.äg. Gau) ${ }^{39}$}

|8| Bist du in Koptos im Haus der Herzensfreude ? - so vereinigt Senut ${ }^{40}$ deine Mumiengestalt. Dein Sohn ist dein Nachfolger als |9| König. Horus ist der Schützer deiner geheimen Gestalt ( $\mathrm{rrw}$ ) im Mumifizierungshaus $(b m ? g)^{44}$.

\section{(4. u.äg. Gau) $)^{34}$}

Bist du im 4. u.äg. Gau, in Djeqa-per ${ }^{35}$, dem Tempel des Hapi-des-Südens ${ }^{36}$ ? - so ist dein Vater $|7|$ neben dir. Er ist der $j m n^{37}$, der deinen Körper reinigt im heiligen $\mathrm{See}^{38}$.

\section{(5. u.äg. Gau $)^{41}$}

Bist du in Sais, im Inneren des $|8|$ Hut-Biti ? - Osiris, komm zu den beiden Rechti ! Die große Achet(-Kuh) ${ }^{42}$, sie macht dich trefflich ${ }^{43}$, sie zieht dich auf ${ }^{45},|9|$ sie vereinigt dich mit der Sonnenscheibe des Atum ${ }^{46}$.

${ }^{26}$ Text B $=$ CD II, 133,1: „Bist du in Fag als Herr auf dem ...-Tempel ? - so hat der Tempel des rechten Auges deine Schönheit." Zum „Auge“ in Text B = CD II, 133,1 s. Beinlich a.a.O. S.12. Das rechte Auge des Re ist Nechbet, s.: RdM V, 63: „Du (Hathor) bist Nechbet, das rechte Auge des Re...“; s.a. E. III, 264,6.

${ }^{27}$ Dümichen hat, ,hw.t-sfj.t".

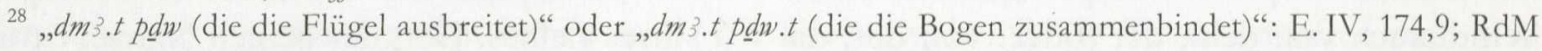
III, 78, E. VII, 301,8; E. V, 197,11.

${ }^{29}$ „Die deine Gestalt geschaffen hat“ könnte hier Tefnut sein, vgl. Philae 89 (VI), RdM V, 4 („Deine Mutter Tefnut ...") u. Opet 211. Die Wortwahl, ,km " $^{*}$ ist wohl durch die Wortspielmöglichkeit mit ,kmj.t = Weihrauchharz" begründet. „k. $m^{3}$ " erscheint noch in E. II, 174 (III), RdM III, 19, RdM V, 63.

${ }^{30}$ Natron ist das typische Produkt des 3. oberägyptischen Gaus, vgl. Beinlich, in: SAK 7, 1979, S. 12 f. Zum Reinigen des Leibes mit Natron s. Tôd, Nr. 84; Opet 211.

${ }^{31}$ Text B = CD II, 134,1: „Bist du in Imaw, der Stadt des Apis? - so schützt der Tempel der Sechat-Hor deinen Körper.“ Zu „Sechat-Hor“ s.: E. IV, 24,5; E. V, 91,15; RdM VI, 109; Philae 114 (VI) („Die Sechat-Hor schützt deinen Körper“c.) vgl. RdM V, 17.

${ }^{32}$ Text B = CD II, 133,1f.: „Bist du in Theben, der Herrin der göttlichen Gaue Ägyptens? - so hast du dort dein Entstehen begonnen." Vgl. dazu CD I, 92,9.

${ }^{33}$ Zum „Geburtsort des Osiris“ s. Philae 89 (VII); RdM V, 5; RdM III, 78 („Er (Amun) gibt den Lebensodem an deine Nase, damit dich deine Mutter gebiert in Theben").

${ }^{34}$ Text B = CD II, 134,3f.: „Bist du im 4. u.äg. Gau, in Djeqa-per ? - so hast du dich niedergelassen in $c h-w r$. Zu „ḩ-w w s.: RdM III, 82; Osirisreliquien, S. 160; Dämonenprozession (MD IV, 62).

${ }^{35} \mathrm{Zu}$ „Djeqa-per“ s.: RdM V, 17; Philae $114 \mathrm{f}$. (VII).

${ }^{36} \mathrm{Zu}$ „Hapi-des-Südens“ s.: E. VI, 24,12f.; RdM VI, 110: E. II, 182,7; Opet 242; Opet 243 (c und d): Opet 190; RdM V, 17; Philae 114f. (VII).

${ }^{37} \mathrm{Zu}$, ,jmn“ (mit Schlange determiniert): E. I, 331,2.

38 „Gereinigt ist dein Körper im heiligen See“: Philae $114 \mathrm{f}$. (VII), vgl. RdM V, 17

39 Text B = CD II, 133,2: „Bist du in Koptos im Haus ...? - so schützt Senut deinen Leib.“

${ }^{40}$ RdM V, 68: „Du (Hathor) bist die Schwester dessen, der sich im Haus der Herzensfreude befindet, die, die ihren Bruder in Senut (?) verjüngt."

${ }^{41}$ S. Médamoud Nr. 147: „... deine geheime Gestalt ist im Mumifizierungshaus. “ Vgl. auch Opet 278: „Du bist der mit schwarzem Leib und gesalbten Gliedern in deinem Namen Osiris vom Mumifizierungshaus."

${ }^{42}$ Text B $=$ CD II, 134,5f.: „Bist du in Sais, im Inneren des Hut-Biti ? - Osiris, komm zu deinem Sohn.“

${ }^{43} \mathrm{Zu}$ „Achet-Kuh“ s.: Opet 244; E. III, 257,9; E. VII, 146,2; CD I, 131.

$44 \mathrm{Zu}$, ,smnb" vgl.: Opet 245

${ }^{45} \mathrm{Zu}$ „rrj“" vgl.: Opet 191; Medamoud Nr. 201.

${ }^{46}$ Zur Nennung des Atum vgl.: Opet 244; E. IV, 25; zu „Sonnenscheibe des Atum“ vgl. Myśliwiec, Atum II, 149 (so nicht dort). 


\section{(6. o.äg. Gau) $)^{47}$}

Bist du in Iunet, im Tempel des Semataui, |10| dem Tempel des Auges [des Re] ${ }^{48}$ ? - so veranlaßt Re die Abwehr gegen deine Feinde. Deine Schwester, die Goldene, ist dein Schutz.

\section{(7. o.äg. Gau $)^{53}$}

Bist du $|11|$ in Kenemet des Sistrum-Hauses ? - so macht das Benu-Haus, was du begehrst ${ }^{54}$. Die beiden Klagefrauen zusammen schützen ${ }^{55}$ deine Majestät gemeinsam. Du fliegst |12| zum Himmel als Benu.

\section{(8. o.äg. Gau $)^{59}$}

Bist du in Ta-wer ? - so hat Abydos deinen $\mathrm{Ba}$, indem du lebst im Sarg. |13| Umhüllt sind deine Sachen durch die Arbeit der Schentit, Horus libiert Kühlung für deinen göttlichen Ka.
(6. u.äg. Gau $)^{49}$

Bist du in Chasut, dem ...-Tempel des Atum ${ }^{50}$ ? - so ist das herrliche Nemset-Gefäß ${ }^{51}|10|$ vollständig mit seinen Dingen. Gereinigt ist [dein] Leib am Platz der Neith. Die Plätze des $\mathrm{Re}^{52}$ haben deine Schönheit.

\section{(7. u.äg. Gau) $)^{56}$}

Bist du in |11| Senetj-nefer im 7. u.äg. Gau ? - so hat Hut-Nehet ${ }^{57}$ deine Furcht. Dein Sohn ist der $m r$-Stier ${ }^{58}$ seiner Mutter. Isis ist der Schutz deines Kultbildes ( $55 \mathrm{~m}$ ) in Genu (?).

\section{(8. u.äg. Gau) $)^{60}$}

|12| Bist du in Pithom, an der östlichen Mündung ? - so bist du lebend als Lebender $(G o t t)^{61}$. Re geht auf und bringt für dich (deine) Dinge, er (?) ${ }^{62}$ läßt sich nieder $|13|$ und schützt deinen Bedarf.

\footnotetext{
${ }^{47}$ Text B = CD II, 133,2f.: „Bist du im Iunet im Tempel des Sema-taui ? - so bin ich neben dir als Harsomtus.“ Betonung des Harsomtus: E. V, 110,12; Mamm. E. 60,5; E. VIII, 6,11; E. IV, 177,2; Opet 212: „neben dir als Harsomtus".

${ }^{48}$ Die Vorstellung von Hathor als dem Auge des Re ist weit verbreitet.

${ }^{49}$ Text B = CD II, 134,4f.: „Bist du in Chasut, dem Haus des Königtums des Re ? - so ist das Verborgene darin der Schutz deines Leibes.“ Vgl. Opet, 191: „Er kommt aus Chasut, dem Haus des Königtums des Re. Das Verborgene darin ( $j m-s$ mit Stadtdeterminativ) ist der Schutz deines Leibes." Zum „Haus des Königtums des Re“ s.: E. VI, 39,5; E. V, 92,12; E. VII, 147,12; Osirisreliquien, S. 166; zum „Verbergen (allerdings jmn)“: E. IV, 25,17; Griffith: Tell el-Yahudiyeh, S. 69; Medamoud, Nr. 203; Opet, 246.

${ }^{50}$ Atum: Opet, 246.

${ }^{51}$ Zum „Nemset-Gefäß“ s.: Opet246f.; Osirisreliquien, S. 166 vgl. S. 245 ff.; RdM V, 18: „das herrliche Nemset-Gefäß ist wohlbehalten ( $\left.{ }^{c} d\right)$ mit seinen Dingen" (vgl. Philae 115 (IX)).

${ }^{52}$ Zum „Platz des Re“ s.: Opet, 247.

${ }^{53}$ Text B = CD II, 133,3: „Bist du in Kenemet des Sistrum-Hauses? - so macht das Benu-Haus, was du begehrst.“

${ }^{54}$ Vgl. Opet 213: „das ...-Haus macht, was du begehrst“. S. auch Ombos 887.

${ }^{55}$ Vgl. CD I, 93: „Geschützt (ḅn) ist der Benu-Vogel im Haus des Benu-Vogels“; vgl. auch E. VII, 307f. Zum „Schützen“ vgl.: Opet 213; „, seine beiden Schwestern zusammen sind ( $m$ ?) sein Schutz“: RdM V, 6; „die beiden Frauen sind dein Schutz": Philae 90 (X) u. RdM V, 6; vgl. E. V, 194,2: „diese beiden Frauen sind sein Schutz“.

${ }^{56}$ Text B $=$ CD II, 134,4: „Bist du in Senetj-nefer im 7. u.äg. Gau ? - so hat Hut-Nehet deinen Respekt ...“ Vgl. dazu Opet 192: „..., um deinen Respekt zu sehen, deine Schwester Isis ist der Schutz deines Leibes“.

${ }^{57}$ Hut-Nehet: „Hut Nehet ist der Schutz $(n h)$ hinter dir“: RdM V, 19 u. Philae $115(\mathrm{X})$; Alliterationen zu Nehet auch MD IV, 61 (nh.t $=$ Schutz, $n$ hs $=$ Seth); Opet 248; E. V, 89,15; E. VII, 149, 6. u. 10; E. III, 257, 13 u. 17; E. III, 255, 16; Nehet (Sykomore): E. I, 331,17-332,1.

${ }^{58}$ „Dein Sohn Horus ist der Stier $\left(k_{3}^{3}\right)$ beim Angreifen ( $\left.b_{3}^{3} j\right)$ seiner Feinde“: RdM V, 19 u. Philae 115 (X).

${ }^{59}$ Text B = CD II, 133,3f.: „Bist du in Thinis, der Stadt des Cheper? - so lebst du in Areq-heh. “ Zum Ausdruck „Stadt des Cheper" vgl.: Tôd, Nr. 89; CD I, 93; Philae 90 (XI), RdM V, 7; RdM V, 73; Opet 213; E. VI, 229,14; zu „Areq-heh"vgl.: RdM V, 7.

${ }^{60}$ Text B = CD II, 131,10: „Bist du in Pithom, an der östlichen Mündung? - so (leb)st du als (Leb)ender (Gott) ...“

${ }^{61}$ Opet 192: „Er kommt aus Pithom, an der östlichen Mündung, lebend als Lebende (Schlange).

$62,-f "$ ? oder Gottesname ? (Atum ?).
} 


\section{(9. o.äg. Gau) $)^{63}$}

Bist $\mathrm{du}^{64}$ in Ipu, dem Platz $|14|$ deines lebenden Fleisches (Phallus ?) ? - so ist Aperet-Iset dein Schutz. Du bist der heiße Stier, der sich verbirgt am Neumond-Fest, der an den Himmel heraustritt am Monatsanfangs-Fest ${ }^{65}$.

(10. o.äg. Gau $)^{68}$

$(\ldots \text { ? })^{69}-|15|$ Du bist (so bist du ?) der Gott, du siehst den Goldhorus, der auf dem Thron der beiden Herren steht ${ }^{70}$. Du hast deinen Sohn erblickt, beim Suchen des Beines. |16| Jubel entsteht auf dem Rücken des Bösen ${ }^{71}$.

\section{(9. u.äg. Gau) ${ }^{66}$}

Bist du in Busiris, im Gebiet des Preisens ${ }^{67}$ ? - so sind diese deine beiden Schwestern dein Schutz. Es kommt deine Gestalt $|14|$ in der Arbeit der Schentit. Die Strahlen des Re vereinigen sich auf deinem Kultbild $(55 \mathrm{~m})$.

\section{(10. u.äg. Gau $)^{72}$}

Bist du in Athribis in Hap-iutiu ${ }^{73}$ ? - |15| so stehst du in der Halle der beiden Wahrheiten. Dein Sohn erhebt seine Stimme nach dem Wunsche deines $\mathrm{Ka}$, und wacht über dein Herz $^{74}$ an [seinem] Platz.

${ }^{63}$ Text B = CD II, 133,4f.: „Bist du in Ipu, verbunden mit (?) der Doppelmaat ? - so schützt Aperet-Iset dein š̌m-Bild.“ S. dazu: RdM V, 75: „Du bist Aperet-Iset...“ u. Opet 214: „Aperet-Iset schützt dein sšm-Bild.“

${ }^{64}, j n$ " fehlt bei Mariette.

${ }^{65}$ Zum „heißen Stier“ in Verbindung mit „,geographischen Inschriften“ in bezug auf den 9. o.äg. Gau s.: E. V, 113,5f.: „Du bist der heiße Stier, der ... am Neumondfest, der an den Himmel heraustritt am Monatsanfangsfest“; Opet 286: „Die Stadt des heißen Stiers“; Opet 214: „Du bist der heiße Stier, der dahin geht am Neumond-Fest, der an den Himmel heraustritt am Monatsanfangs-Fest“; Ombos Nr. 888: „Du bist der heiße Stier, der gemacht hat $(? j r j-n-f)$ das Neumond-Fest, der du heraustrittst zum (? $n$ ) Himmel am Monatsanfangs-Fest in deinem Namen

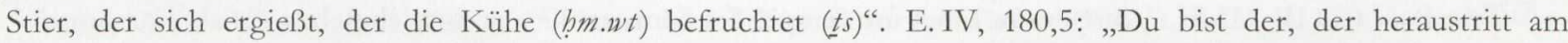
Monatsanfangs-Fest (tp dnj.t)... (vgl. Medamoud, Nr. 171)“; E. VII, 311,11f.: „Min ... der erste im Haus des Mondes, der heraustritt am Tage des Neumond-Festes, Stellvertreter des Mondes“; Gauthier, Les fêtes du dieu Min, S. 29 u. 72: „der herausgeht aus den beiden Sanktuaren zum Neumond-Fest als Stellvertreter des heißen Stieres“; E. VII, 311,8: „Stier, Herr des Monatsanfangs-Festes“.

${ }^{66}$ Text B = CD II, 134,3: „Bist du in Busiris, im Gebiet des Preises? - so ist Anedjti überschwemmt mit deinem jr.w-Bild.“ Vgl. dazu Opet 193: „Er kommt aus Busiris, aus dem Inneren des Gebietes des Preisens, Anedjti ist überschwemmt mit seinem (des Anedjti als Name des $m$ r-Kanals und des Gottes ?) jr.w-Bild."

${ }^{67}$ Zum „Gebiet des Preisens“ s.: E. III, 247,14; E. VIII, 120,3f.

${ }^{68}$ Text B = CD II, 133,5: „Ich bin in *Tjebti, ich bin der Löwe, die Feinde gegen mich sind in ... (ndn? ntt-Fessel ?). “Zum Löwen s.: E. V, 113,14f.; Mamm. E. 60,17; Medamoud Nr. 174; Opet 288; Opet 214; Osirisreliquien, S. 116.

${ }^{69}$ Als einzigem der aufgezählten Orte fehlt dem 10. o.äg. Gau die einleitende Frage. Dies könnte ein Versehen sein, so daß der Text fortfahren müßte „... so bist du ...", es könnte aber auch Absicht sein, wodurch dem 10. o.äg. Gau eine Sonderstellung gegenüber den anderen Gauen verliehen würde.

${ }^{70}$ Vgl.: E. IV, 181,2: „Du bist der Goldhorus, der den Thron der beiden Herren ergreift“; vgl. dazu auch Medamoud Nr. 174 und E. VII, 313,11.

71, ,dw" ist wegen der Ortsbezeichnung , „dw-k $w_{3}^{3}$ (Qau el-Kebir)“ häufig Ausgangspunkt für ein Wortspiel, vgl.: E. IV, 182,1; Medamoud Nr. 175; E. VII, 313,2 („sw $\underline{\text { d }}$ d.t-k $\left.r \underline{d} w^{\prime \prime}\right)$; Osirisreliquien, S. 116: „Ich bringe eilends den Oberschenkel vom Gebirge ( $\underline{d} w)$, ich bringe ihn zu seinem Herrn, sein Herz ist in Freude “ CD I, 94: „... jubelnd auf dem Rücken des Bösen $(\underline{d} w)^{\text {“; }}$ RdM V, 78: „Du bist die Herrscherin, die ihren Sohn sieht in Jubel auf dem

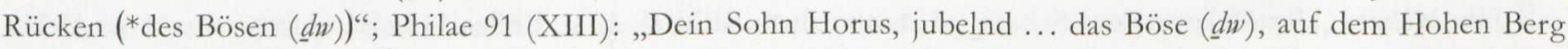
$\left(\underline{d} w-k_{3}^{3}\right)^{\text {“; }}$ RdM III, 8: „Dein Sohn Horus, der jubelt wenn er dich sieht, der vertrieben hat das Böse auf dem „Hohen $\operatorname{Berg}(\underline{d} w-\underline{k}, 3)^{“} ; \operatorname{RdM~V,~77:~„Du~bist~die~Antit,~die~abwendet~den~Schritt~des~Bösartigen~(?~ked~dw~?)“;~E.~VII,~6,13:~}$ „Du bist der, der etwas sucht auf dem Berg ( $\underline{d} w)$ (von ?) Wadjet, was vollendet ist im Inneren von Ra-qereret“; Osirisreliquien, S. 116: „der Erbe des Iun, der das Bein seines Vaters sucht“.

${ }^{72}$ Text B = CD II, 134,2f.: „Bist du in Athribis ... (2. Quadrate) in Hap-iutiu ? - so siehst du die Gestalt deines Sohnes (*so siehst du, was dein Sohn tut)." S. dazu: Vernus, Athribis, S. $298 \mathrm{ff}$. u. 423, 436. Zu "*so siehst du, was dein Sohn tut"vgl. Opet, 193; Philae 116 (XIII); RdM V, 20; Vernus, Athribis, S. 301.

${ }^{73}$ Osirisreliquien, 174; Vernus, Athribis, S. 265 (Taf. 44); E. V, 9317.

${ }^{74}$ Zum „Herzen“ s. Vernus, Athribis, S. 433 ff.; Beinlich, Osirisreliquien, S. 255 f. 


\section{(11. o.äg. Gau) ${ }^{75}$}

Bist du in Schashotep im Inneren des Hutnememet $^{76}$ ? - so wünscht man nicht, daß |17| man dein Kultbild ( $s s^{\llcorner} m$ ) sieht. Dein Vater Geb umwickelt $^{77}$ deine Glieder, verborgen wird dein Leib durch Anubis ${ }^{78}$.

\section{(12. o.äg. Gau $)^{79}$}

Bist du in |18| Per-Anti, der Stadt des Falken ? - so bist du zufrieden in „Vereinigung der Freude ${ }^{\text {c80 }}$. Dein Sohn ist der [lebende] Falke auf dem $\operatorname{Kopf}^{81}$ des Schlachtrindes ${ }^{82}$. Nicht kann das Böse herankommen zu deinem Körper.

\section{(13. o.äg. Gau $)^{88}$}

Bist du $|19|$ in Assiut, dem Haus deines Gottesleibes? - so ist dein Mysterium verborgen am Platz des Vereinigens. Geöffnet ist dir dein Mund $^{89}$ durch den oberägyptischen Upuaut. Deine beiden Uräen $(n b . t j){ }^{90}$ sind dein Schutz.

\section{(11. u.äg. Gau)}

Bist du $|16|$ in Schedenu an der Spitze der lebenden (Götter) ? - so erstrahlst du als Herr von Meret. Der, der in Ta-wer ist, ist gesund in der Mesektet-Barke. |17| Sein Schutz sind die Götter von Schedenu.

\section{(12. u.äg. Gau) $)^{83}$}

Bist du in Tjeb-netjeret, dem Tempel des Heri-ideb $^{84}$ (Schu) ? - so ist dein Vater hinter dir als Herr des Maba-Speers ${ }^{85}$. |18| Deine Mutter Djedet ist beim Verbrennen ${ }^{86}$ deiner Feinde $(\underline{b} \supset k-j b w)$. Deine Nasenlöcher ( $\underline{b} n m . t j-k)$ empfangen den Atem (srk- k. ptjjt) $)^{87}$.

\section{(13. u.äg. Gau $)^{91}$}

Bist du in Heliopolis, deinem „Himmel ${ }^{\star 692}$ ? so läßt du dich nieder in Uha-ta ${ }^{93}$. Du kommst $|19|$ als Ba und als Benu zu deiner geheimen Gestalt (bs) beim Sprechen der Klagen der beiden Klagefrauen (Isis u. Nephthys).

\footnotetext{
${ }^{75}$ Text B $=$ CD II, 133,5f.: „Bist du in Schashotep im Inneren des Hut-nememet ? - so wünscht man nicht, daß man dein Kultbild ( $5 \check{m} m$ ) sieht."

${ }^{76} \mathrm{Zu}$ „Hut-nememet“ s.: Philae 91 (XIV) (3×); E. I, 340,5; Nememet-Bahre: E. VIII, 6,14; wahrscheinlich besteht eine Wortbeziehung zum Nememet-Gebiet: E. IV, 182,9 ff.; E. V, 115,3ff.; RdM V, 80.

77 „Umwickeln - nwj“? (WB IV, 341.16 gibt als Lesung „stp“. Der einzige Beleg (E. I, 183,16) zeigt aber „nwj“).

${ }^{78}$ Evtl. gibt es eine Verbindung zwischen dem Einwickeln und Verbergen einerseits und dem Opfer des Stoffes (E. V, 190) in Verbindung mit dem Gau und dem spezifischen Gauopfer (st3m-Kleidung) andererseits. Vgl. Beinlich, in: SAK 7, 1979, S. 16.

${ }^{79}$ Text B = CD II, 130,11: „Bist du in Per-Anti, der Stadt des Falken ? - so bist du zufrieden in ,Vereinigung der Freude“."

${ }^{80} \mathrm{Zu}$ „Vereinigung der Freude“ s.: Philae 91 (XV); RdM V, 9; RdM V, 81; E. I, 340,11 (z. T. zerstört).

${ }^{81}$ Philae 91 (XV): „Dein Sohn Horus ist auf dem Kopf der Feinde“ (vgl.: RdM V, 82; vgl. E. I, 340,10f.). Die Vorstellung von Horus auf dem Kopf des Feindes ist deutlich beeinflußt von der Hieroglyphe G $201 \mathrm{ff}$. (Valeurs phonétiques ...), die den Falken auf einem Rinderkopf oder einem ähnlichen Gegenstand zeigt.

${ }^{82}$ E. VIII, 6,14: „Du bist der Falke, der auf seinen Klauen steht, der das Schlachtrind tötet mit seinen Krallen“; E. III, 276,16 (vgl. auch 278,10f. u. E. VII, 316,14f.): „Der Falke ..., der auf dem Kopf seiner Feinde steht“. Zu dieser Vorstellung von Anti auf dem Kopf des besiegten Tieres gehört auch das spezifische Gauritual: „Schlachten des Schlachtrindes" (E. VII, 316) bzw. „ *Schlachten der Antilope“ (E. III, 278 Titel zerstört). Vgl. Beinlich, in: SAK 7, 1979, S. 21.

${ }^{83}$ Text B = CD II, 132,3f.: „Bist du in Tjeb-netjeret, dem Tempel des Heri-ideb (Schu) ? - so sind deine Feinde getötet in Hut-(biti ?)-Hor.“ Vgl. dazu Opet 194 „Seine Feinde sind getötet“.

${ }^{84} \mathrm{Vgl}$. Opet 194.

${ }^{85}$ Zum „Maba-Speer“ s.: E. IV, 31,5; E. VII, 159,5 u. 9; E. III, 246,7; Philae 117 (XV) u. RdM V, 21 („Schu ist hinter dir als Herr des Maba-Speeres“).

${ }^{86}$ Opet 194: „Deine Mutter Tefnut verbrennt $(w b d)$ deine Feinde.“

87 Opet 194: „Du bist der Lufthauch $(j s-n j w)$ in den Nasenlöchern, der Herr des Lebens und des Atems $(s r k$ - $b t j j t) “$, vgl. auch E. IV, 31,8; zum „Windhauch“s. auch Philae 117 (XV) u. RdM V, 21.

${ }^{88}$ Text B $=$ CD II, 130,11 f.: „Bist du in Assiut, dem Haus deines Gottesleibes ? - so ist dein Mysterium verborgen am Platz des Vereinigens.“
} 
(14. o.äg. Gau $)^{94}$

$|20|$ Bist du in Kusae, deiner göttlichen Kapelle, dem Tempel der Herrin der Götter (Hathor)? - so ist dein Ausfluß geschützt durch Kebehsenuef $^{95}$. Dein Feind ${ }^{96}$ ist gefesselt ${ }^{97} \ldots|21|$ seine Fessel ${ }^{98}$.

\section{(15. o.äg. Gau $)^{102}$}

Bist du in Unut, dem Platz des Erschaffens des Lichtes ${ }^{103}$ ? - so hat der Tempel ${ }^{104}$ der Maat deine Vollkommenheit $(n f r w)^{105}$. Gefangen $(* j b \underline{t})$ ist Seth neben dem Hut-ibet ${ }^{106}$ des Chenti-mekes.

(16. o.äg. Gau $)^{110}$

|22| Bist du in Hebenu im Haus des Triumphes $^{111}\left(m_{3}^{{ }^{c}}-b r w\right)$ ? - so bist du zufrieden als Herr (des Ortes) „Herzensweite“112. Dein Sohn, der Held, er hat Böses dem angetan, der (es) ihm angetan hat. Er hat zurückgetrieben ${ }^{113}$ die Feinde in $|23|$ ihren Fesseln ${ }^{114}$. Die in Sa-ka ${ }^{115}$ sind gefallen im Hause des Zerhackens ( $p r$ - bbj) beim Gemetzel dessen, der erhaben an Arm ist... gegeben ist $\left(r d j\right.$ ?) der Feind $(s n t j)^{116}$ unter seinen Herrn. Er hat gewendet das Übel gegen den, der es getan hat.
(15. u.äg. Gau $)^{99}$

Bist du in Bah, im Hut-meriti ? - so läßt Thot $^{100}$ dich triumphieren. Er macht dein Herz fest. $|20|$ Er führt für dich die Mundöffnung aus. Er macht deinen Spruch (?) richtig ${ }^{101}$ vor deinem Thron.

(16. u.äg. Gau $)^{107}$

Bist du in Mendes, im Haus der Götterneunheit $^{108}$ ? - so jauchzen die beiden Schwestern, wenn sie dich sehen. Der Duft des Gotteslandes $(?)^{109}$ durchzieht ( $p \underline{b} r$ ? ) deine Wohnung (jwnn). |21| Dein Ka verjüngt sich als Widder.

(14. u.äg. Gau $)^{117}$

Bist du in Tjaru, in Chent-iabet? - so hat das nördliche Djeba deine Vollkommenheit. Du bist der (Sonnen)-Käfer $\left({ }^{c} b b\right)^{118}$, der zuerst herausgekommen ist in Ta-wer. $|22|$ Dein Sohn ist der Hüter ${ }^{119}$ der Tür von Ägypten.

${ }^{89}$ Zur Beziehung zwischen „Mund(öffnen)“ und „Upuaut von Assiut“ vgl. auch Sauneron, Rituel de
l'Embaumement 4. $23 \mathrm{f}$.
${ }^{90}$ Vgl. E. V, 116,12: „... die beiden Herrinnen (nb.tj) an seiner Spitze“. Zu den beiden Uräen vgl. auch Opet, 293; E. I, 341,3; Philae 91 f. (XVI); Mamm. E. 81,12-16; E. V, 188,17; E. III, 280,17 u. 281,13; pLouvre I 3079, Z. 77: Goyon, in: BIFAO 65, 1967, S. 151 u. 105.

91 Text B = CD II, 134,6: „Bist du in Heliopolis, dem „Himmel von Ägypten“ ? - so läßt du dich nieder ... “

${ }^{92}$ Vgl. zu diesem Ausdruck: RdM VI, 119: „... die sich mit ihrem Vater vereinigt im ,Himmel-von-Ägypten““, Opet 194: zu „Heliopolis, ,Himmel von Ägypten““, vgl. auch E. V, 95,7 u. MD IV, 62.

${ }^{93} \mathrm{Zu}$ „Uha-ta“ s.: Gauthier, DG I, 203; RdM VI, 119; E. VII, 166,17.

${ }^{94}$ Text B = CD II, 130,12: „Bist du in Kusae, deiner göttlichen Kapelle? - so ist der Schutz deines Ausflusses (Kebehsen)uef."

${ }^{95} \mathrm{Zu} \mathrm{Kebehsenuef} \mathrm{und} \mathrm{dem} \mathrm{Ausfluß} \mathrm{s.:} \mathrm{E.} \mathrm{I,} \mathrm{341;} \mathrm{vgl.} \mathrm{Osirisreliquien,} \mathrm{S.} 51$ u. 124.

96 „Dein Feind“ fehlt bei Dümichen.

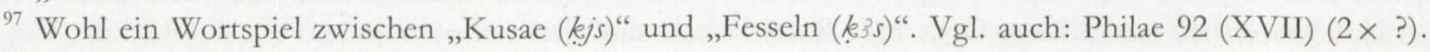

${ }^{98} \mathrm{Zu}$, ,ntt. vgl.: RdM V, 86.

99 Tंext B = CD II, 132,2f.: „Bist du in der Thot-Stadt, im Hut-meriti? - so bist du dauerhaft im Per-wenech.“ Zu „Per-wenech“ vgl.: RdM VI, 121; Medamoud, Nr. 229; E. V, 91,1; E. III, 251, 13; (Derchain-Urtel, Thot, S. 12 , $151 \mathrm{f}$.$) .$

${ }^{100}, g s . t j "=$ Palette.

${ }^{101}$ Vgl. Seeber, Totengericht, S. 152. Zu ,sm $3^{c}-b r w^{\prime \prime}$ vgl.: RdM VI, 121.

102 Text B = CD II, 130,12-131,1: „Bist du in Hermopolis, dem ersten Gau, in dem das Licht angefangen hat? - so hat der Tempel der Maat deine Vollkommenheit (nfrw).“ 
(18. o.äg. Gau) $)^{120}$

|24| Bist du in Anti (Dunanui ?) im Inneren der Gotteshalle ${ }^{121}$ ? - so siehst du deinen Sohn als Iunmutef ${ }^{122}$. Horus ist es, der seine Flügel ausbreitet ( $h d j)$ beim Auffliegen ${ }^{123}$. Er hat gepackt die Erde als Eigentum seines Vaters.

\section{(20. o.äg. Gau $)^{126}$}

|25| Bist du in Herakleopolis, in den beiden südlichen Heiligtümern ${ }^{127}$ ? - so ist deinem Kopf die Atef-Krone ${ }^{128}$ aufgesetzt. Geschützt ist deine Gestalt in Hebes-bega ${ }^{129}$. Heneb ${ }^{130}$ schützt deinen Körper.
(17. u.äg. Gau) $)^{124}$

Bist du in Sema-behedet, dem Platz des Vereinigens der beiden Länder? - so bist du geschützt als Cheper-djes-ef ${ }^{125}$. Du bist Osiris, |23| indem er machtvoll ist in seinem Bild. Die Angelegenheiten des Landes sind deinem Willen unterworfen ( $\underline{b} r$ s.t $r_{3}$-ke).

\section{(18. u.äg. Gau $)^{131}$}

Bist du in Netjer, in Us-em-pet ${ }^{132}$ ? - so sind die Bau $|24|$ der Götter und Göttinnen im Gefolge deines Ka. Wat (wว.t) Heqat (bk.j.t) gesellt sich zu dir täglich. Sie hat herrlich gemacht (? $\underline{d} s r$ ) die Beiden Länder unter (mit ? $\underline{b r}$ ) deinem Körper. Die Mehenet-Schlange des Re ist zugeordnet deiner Kraft im Leben. |25| Sie richtet ihren Atem ${ }^{133}$ gegen deine Feinde.

\footnotetext{
${ }^{103}$ Vgl. dazu: E. V, 118,9 u. Opet 297. S. auch „der hohe Hügel (Urhügel) an dem das Licht angefangen hat“: RdM V, 10 und Philae 92 (XVIII).

${ }^{104}$ Die Kuh in der Tempelhieroglyphe könnte eine Anspielung auf Mehet-weret sein.

${ }^{105}$ In der Verbindung mit dem Gauzeichen könnte „nfrw" eine Anspielung auf Wenennefer sein.

${ }^{106} \mathrm{Vgl}$. zu „Hut-ibetj“: E.I, 341,12; E. VII, 322,11; E. V, 187,7. Es ist ganz eindeutig so, daß die inhaltliche Aussage, daß Seth gefangen sei, sich an dem vorgegebenen Ortsnamen orientiert.

107 Text B = CD II, 132,3: „Bist du in Mendes, im Haus der Götterneunheit ? - so jauchzt deine Majestät über die Tätigkeit (k3.t) der Frauen. " Es ist möglich, daß bei k3.t etwas Konkreteres aus dem sexuellen Bereich gemeint ist, vgl. etwa Kurth, Dekoration der Säulen, GOF IV, 11, S. 152f. u. Anm. 2.

${ }^{108}$ Vgl.: E. VI, 41,7.

${ }^{109}$ Evtl. auch nur „der Gottesduft“, dann sind aber die Zeichenreste nicht untergebracht.

${ }^{110}$ Text B $=$ CD II, 131,1 f.: „Bist du in Hebenu im Haus des Triumphes $\left(m^{c}{ }^{c}-b r w\right)$ ? - so bist du zufrieden als Herr der Herzensweite. “

${ }^{111}$ Die Vorstellung vom „Haus des Triumphes“ ist beeinflußt vom Gauzeichen des Falken auf der Antilope (s. dazu Osirisreliquien, S. 232f.). Vgl. auch E. V, 118,13ff.; Mamm. E. 61,15ff. u. Opet 298; s. auch Philae 92 (XIX) u. RdM V, 11; RdM V, 90; E. V, $186 f$.

${ }^{112}$ Vgl. Philae 92 (XIX) u. RdM V, 11.

${ }^{113}$ Wortspiel „,hbnw - ḥnb (zurücktreiben)“; vgl. auch das Wortspiel CD I, 95: „ḥnb - bḥn" („,.. sie hat zurückgetrieben $(\underline{h n b})$ den Seth $(b)$, geschlachtet $(b \not ̣ n)$ ist der Feind (šntj) wegen (?) dessen, was er gemacht hat.") Vgl. auch RdM V, 89 (rechts); zu „bhnn“s. auch RdM V, 90 (rechts) u. im Zusammenhang mit dem 17. o.äg. Gau RdM V, 92 u. Opet 300.

${ }^{114} \mathrm{Zu}$, ,ntt" vgl. auch Ombos 892 (,... gefesselt in seiner Fesselung“); RdM V, 92.

${ }^{115}$ Der fehlende 17. o.äg. Gau ist durch die Nennung des typhonischen Sa-ka vertreten. Vgl. Ombos 892, E. VI, 212,2; Philae 93 (XX), RdM V, 11; Opet 300 f.; E. VI, 231,1; RdM III, 81. Möglicherweise hängt die Nennung des šntj mit dem Gebiet von Sa-ka zusammen, denn dieser Begriff erscheint mehrfach in Verbindung mit dem 17. o.äg. Gau: Ombos 892, CD I, 95.

${ }^{116} \mathrm{Vgl}$. CD I, 95.

117 Text B = CD II, 132,1 f.: „Bist du in Tjaru, an den Horuswegen ? - so ... ersinnen gegen deine Majetät.“ Man erwartet hier eigentlich, daß es ,nichts gibt, was“, oder „niemanden gibt, der“ „etwas gegen deine Majestät ersinnt“. Sollten die beiden „מ-Schleifen“, die man in der Textausgabe sieht, „nn“ zu lesen sein (2 nw-Töpfe ?) ? „Es gibt kein Machtwesen, das ...“?

${ }^{118}$ Vgl. E. IV, 33,11; Medamoud, Nr. 228; RdM VI, $120(2 \times)$.

${ }^{119}$ Vgl. E. V, 102,2.
} 
(21. o.äg. Gau) $)^{134}$

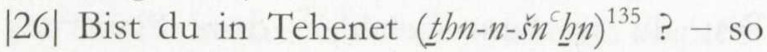
bist du geformt in Neb-irt ${ }^{136}$. Der einzigartige Ima-Baum $\left(j m^{3}-w^{c} t j\right)^{137}$ ist heil ( $(\underline{d})$, ohne Übel, gesund $(2 \times)$ in Ewigkeit.

\section{(22. o.äg. Gau) $)^{141}$}

Bist du $|27|$ in Teh-ihet, im Inneren des Per-wer ? - so schützt die Goldene deine Glieder. Hesat ${ }^{142}$, die Gottesmutter, erstrahlt auf ihrem Thron. Die Tochter des Vaters deines Vaters (?), sie wiederholt die Verjüngung.
(19. u.äg. Gau) ${ }^{138}$

Bist du in Imti-pehu, dem Platz Sched-suwadjet $^{139}$ ? - so ist dein ... ( $\ldots d w$ ? $)$ geschmückt (st ? ?) im Tempel des Seker ${ }^{140}$. - Du bist der erhabene (stnj) $|26|$ an Gestalt, bekleidet $\left(s^{c} h\right)$ mit der Weißen Krone, der Schöngesichtige, der Geliebte der Götter.

\section{(20. u.äg. Gau $)^{143}$}

Bist du in Per-Sopdu, im Inneren des Hauses der Rechtfertigung ? - so bist du verborgen in Imen-Cheperu ${ }^{144}$. Komm zu deinem Sohn, $|27|$ dem dieses Land dient. Erfreue das Herz des Horus und der Mut. Sie fesseln die Feinde an das Tor der Balsamierungs[halle] (wrj.t). Sie hacken die Erde auf ${ }^{145}$ mit ihrem Blut.

${ }^{120}$ Text B $=$ CD II, 131,2f.: „Bist du in Anti (Dunanui ?) im Inneren der Gotteshalle ? - so siehst du auf mich als Iunmutef. Ich bin der Fekti-Priester, der seinen Vater täglich erhaben macht, ohne daß ich müde werde, seine Feinde zu schlagen. Oxyrhynchos ist niedergeworfen, seine Bewohner sind im Feuer, die Habgierigen (die Einwohner der Stadt) sind geschlachtet in der Glut. “

${ }^{121}$ Zur „Gotteshalle“ s.: E. VII, 326,6; E. I, 342,11.

${ }^{122}$ Im Text Philae 93 (XXI) führt der Iunmutef die Mundöffnung durch. Die Mundöffnung ist das Gauritual bei E. III, 286,6, E. VII, 325 f. u. Dendera (unpubliziert), s. Beinlich, in: SAK 7, 1979, S. 21. S. a. Opet 303 (Fragment).

${ }^{123}$ Flügel ausbreiten beim Auffliegen: E. IV, 188,4; Philae 93 (XXI); RdM V, 12; E. III, 286,12; E. VIII, 7,3f.; Flügel (Arme) ausbreiten beim Schützen: RdM III, 81; Philae 93 (XXI); RdM III, 81 (zerstört); E. V, 186,5; Bezug zwischen dem Fliegen und dem Gauzeichen: E. V, 120,8f.; Mamm. E. 62,5; Ombos 893; Opet 302; E. III, 285,14 .

${ }^{124}$ Text B = CD II, 132,4f.: „Bist du in Sema-behedet, dem Platz des Vereinigens der beiden Länder ? - so ist vereinigt der große Gott in $b s j-m^{3^{c}} . t j$ (? - sonst nicht bekannter Ort). “

${ }^{125} \mathrm{Zu}$ „Cheper-djes-ef“ s.: Medamoud, Nr. 238; E. III, 237,3; E. I, 334,16.

${ }^{126}$ Text B = CD II, 131,3f.: „Bist du in Herakleopolis, in den beiden südlichen Heiligtümern ? - so ist deinem Kopf die große Atef-Krone aufgesetzt."

${ }^{127} \mathrm{Zu}$ den „beiden südlichen Heiligtümern“ s.: RdM V, 97.

${ }^{128}$ Zur „Atef-Krone“ s.: Mamm. E. 62,12; E. V, 122,2 (z. T. zerstört); RdM V, 13 („Die Atef-Krone des Re ist auf deinem Kopf “), ähnlich wohl Philae 93 (XXII) (zerstört); RdM III, 81; E. III, 285,15.

$129 \mathrm{Zu}$ „Hebes-bega“ s.: Gauthier, DG 4, 26.

${ }^{130} \mathrm{Zu}$ „Heneb“ s.: E. I, 343,7; RdM V, 13 („Heneb ist dein Schutz“), Philae 93 (XXII).

${ }^{131}$ Text B = CD II, 131,9: „Bist du in ... in Us-em-pet (ws-m-p.t) ? - so schützt Scha-en-bastet (Gauthier, DG 5, 104) deine Dinge." Zu „Scha-en-bastet" s. Ombos II, 871.

${ }^{132} \mathrm{Zu}$, ,ws-m-p.t“ s.: Gauthier, DG I, 205.

${ }^{133}$ Zum „(feurigen ?) Atem gegen die Feinde“ vgl. evtl. E. IV, 36,7 u. Medamoud, Nr. 241.

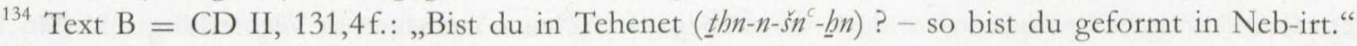

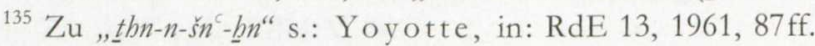

${ }^{136} \mathrm{Zu}$,ked (nbj ?) ... m-bnt nb-jr.t" vgl.: E. I, 343,11; zum Formen vgl.: RdM V, 13 u. Philae 93 (XXIII); Yoyotte, a.a.O., S. $87 \mathrm{f}$.

${ }^{137}$ Zum „Ima-Baum“ s. unten Z. W-33. S. dazu: Yoyotte, in: RdE 13, 1961, 87.

${ }^{138}$ Text B = CD II, 132,1: „(Bist du in ...), dem Platz Sched-su-wadjet ? - so jubelst du in der Wüste des Jubelns.“ Zur „Wüste des Jubelns“ s.: Brugsch, DG 481, 1089 f.; Gauthier, DG 4, S. 16 u. S. 159 ; pLouvre 3079 (Goyon, in: BIFAO 65, 1967, S. 154, Z.93/94, Anm. S. 135); E. V, 99,6; E. VII, 165,9; E. III, $241,5$.

${ }^{139} \mathrm{Zu}$ „Sched-su-wadjet“ s.: E. I, 335,16; (vgl. auch Mamm. E. 67, 17 „śd sw mw.t-f“). 
|28| Bist du in diesen Städten deines Ka ? so haben die Gaue der Götter auf Dauer dein Kultbild $(s \breve{s})$ ). Die Heiligtümer ( $h m w)$ der Machtwesen $(s b m w)$ ermangeln nicht deines lebenden Abbildes $(\mathrm{sbm})$, indem du lebst in Ewigkeit (nḥh).

|29| Der Süden ist unter deiner Hoheit, der Osten ist unter deinem Respekt.

Deine Liebe durchläuft ihre Herzen.

Der Himmel hat deinen Ba.

Die Sterne sind in deinem Gefolge. Deine göttliche Schwester Sothis ist bei deinem Schreiten $^{148}$. Müde ist für dich das Überschwemmungswasser, um die beiden Länder mit Nahrung zu versehen ( $s \underline{d} f_{3}$ ), $|30|$ um für dich die Überschwemmung auszugießen (3x).

Beschenkt ist für dich der Opfertisch mit Brot, Fleisch und Wein, um [deinen] $\mathrm{Ba} z u$ den Opferspeisen zu bringen.

Du bist ja der Gott, der Fürst der Götter,

der älteste Sohn des Iri-pat (Geb).
|28| Bist du in den Stätten der ganzen Erde ( $t_{3}$ ḩr ndb-f) - so haben die Heiligtümer (jtr.tj) der Götter (dein) Bild (twt), die Heiligtümer $(g s-p r)$ deiner Götterneunheit haben dein (bntj-Bild indem du gesund bist $(s n b)$ in Ewigkeit (․․t).

|29| Der Norden ist unter deinem Schrecken, der Westen ist unter deinem Zittern.

Sie geben für dich die Hand auf die Brust ${ }^{146}$ (?)

Die Dat ist schön ist mit deinem schönen $(\underline{d} s r)$ Körper ${ }^{147}$. Deine beiden Djerti sind $|30|$ hinter dir als dein Schutz.

Die ...-Figuren ${ }^{149}$ und die ...-Wesen (?) ${ }^{150}$ sind in deinem Gefolge zusammen. Sie küssen die Erde am Eingang deines Tempels.

Für dich kommt das Gebiet der Berge als göttliche Gesteine herein, damit |31| du trägst deinen $\mathrm{Ka}$ (deine Kraft des ?) ... ${ }^{151}$ ?

Du bist ja (ntk) der göttliche Gott, Herrscher der Tempel,

goldenes Abbild der Nut (Sohn der Nut, vgl. Wb II, 71,6).

\footnotetext{
$140 *$ sgr-Schweigen? *sker-Sokar?, GDG 4, 131; Brugsch, DG 1093f. u. 1330. In der Abschrift von Dümichen hat das Determinativ „Mann“ die Hand am Mund, bei Mariette nicht.

${ }^{141}$ Text B = CD II, 131,5 f.: „Bist du in Tep-ihet, im Inneren des Per-wer ? - so schützt Hathor deine Glieder. Der 22. o.äg. Gau sticht, um niederzuwerfen den ... (Seth), um zufrieden zu machen das Herz des Osiris ..."

${ }^{142} \mathrm{Zu}$ „Hesat“ vgl.: Ombos 895; E. IV, 193,3; RdM VI, 101; E. III, 286,3 u. 287,16.

${ }^{143}$ Text B = CD II, 131,9f.: „Bist du in Per-Sopdu, im Inneren des Hauses der Rechtfertigung ? - so nimmst du Gestalt an in Imen-Cheperu.“

${ }^{144} \mathrm{Zu}$ „Imn-Cheperu“ s.: Gauthier, DG 1, S. 74; RdM V, 25; vgl. Medamoud, Nr. 248 u. Ombos II, 871.

${ }^{145}$ Sie düngen die Erde mit dem Blut der Feinde, vgl. WB III, 256,2 u. TB 18.

146 „mnd", mit diesem Determinativ nicht im WB. Gemeint ist wohl eine Geste, die Liebe und Vertrauen ausdrückt, und wo die Hand auf's Herz gelegt wird.

${ }^{147}$ "tjt“, hier im Gegensatz zu Ba, also der „unbeseelte“ Körper. Zum Begriff vgl. aber auch Ock in ga, S. $101 \mathrm{ff}$.

${ }^{148}$ Sothis als Begleitung des Überschwemmungswassers?

${ }^{149}$ Das erste Zeichen, ein kniender Mann, hält nach Mariette ein Beil an seinem Kopf (Feind), nach Dümichen gießt er Wasser aus einer Hes-Vase über sich (so m. E. auch im Original).

${ }^{150}$ Evtl. auch „die trefflichen (mnb) ....-Figuren“.

${ }^{151}$ Das meint doch wohl die Versorgung mit Amuletten.
} 
Deine Verpflichtungen mögen dauern $|31|$ ewig Deine Bestimmungen mögen dauern ewig (ㅁ.t). $(n \not h b)$.

Sie werden neu (?) zu deinem Erscheinungs-Fest (?). Sein Nichtkennen gibt es nicht.

Deine Tempel $^{152}$ sind so dauerhaft, wie die Sonnenscheibe ${ }^{153}$ dauert. Es gibt kein Vergehen deiner Feste für den Durchführenden.

Wie schön $\left({ }^{c} n\right)$ ist dein Gesicht ${ }^{154}$.

Wie süß $(n \underline{d} m)$ sind deine beiden Lippen $(s p . t j)$. Dein Herz kommt |32| als göttliche Nahrung.

$\mathrm{Du}$ wandelst im Sonnenglanz ( $n d \underline{d} w)$. Du schreitest (bnd) am Abend.

Mögest du hören die Stimme deines Sohnes, wenn er dich preist.

Möge dein Ruheplatz (k. hot) auf dem Serech (Palastfassade, Thron) sein.

Es küssen dich „die Götter, die um ihre Kapelle herum sind $\left(b^{3} w-k{ }^{3} r-s n\right)^{“} \ldots$

|33| Mögest du hören den Jubelgesang des $s_{3}$ - $t_{3}$ auf der Erde $\left(m t_{3}\right)$.

Der herrliche Isched-Baum ${ }^{156}$, mögest du einnehmen deinen Platz neben ihm. Angenehm ist es deinem Körper in seinem Schatten.
... (bm-k $n f$ ?) ... Verjüngung der Vorschrift(en) für deinen $\mathrm{Ka}$, sie erneuern sich.

Dein Sitz, |32| dauert (?) bis zur Ewigkeit des Mondes. Es gibt keinen Mangel für den, der deinen Namen nennt $(d m-r n-k)$.

Wie schön $(n f r)$ ist es, dich zu sehen, Licht (? hdd ), Herr der weißen Krone.

Wie süß (bnr) ist das, was heraustritt aus deinem Munde.

Du schreitest in der Dat, vollständig an deinem Körper.

|33| Mögest du hören die Stimme deiner Djerti, der Schwestern (Isis und Nephthys).

Mögest du besteigen den Thron.

Es preisen dich die Bau von Heliopolis.

Freudig sei [dein] Ka am göttlichen Morgen ${ }^{155}$.

Der einzigartige Ima-Baum ${ }^{157}$, mögest du dich nicht entfernen $|34|$ von $(r-\not ̣ n)$ ihm. Es kommt dein Herz in Jubel.

${ }^{152}$ Bei „,hw.wt" ist offensichtlich das nțr vergessen worden (vgl. M.D. IV, 64, unten rechts u. oben rechts u. CD II, 153,1) oder war nicht notwendig, vgl. auch Z. W-31.

${ }^{153}$ Evtl. ist mit Iten auch die Mondscheibe gemeint. Vgl. etwa MD IV, 68 oben, Z. 7. Dann wäre die Nennung des Mondes auf der Ostwand kein Gegensatz, sondern eine parallele Aussage.

${ }^{154}$ Evtl. handelt es sich hier um den Beginn eines Liedes. S. dazu pN. 3176 (S), VI,7 (Ba rguet, Le papyrus N. $3176($ S), BdE 37, 1962, S. 20).

${ }^{155}$ S. Anm. 156.

${ }^{156}$ Der Ausdruck ,göttlicher Morgen“ gehört in den Sokarbereich. S. dazu: Alliot, Culte d'Horus (BdE 20), S. 794 u. 798f.; Goyon, in: BIFAO 78, 1978, S. 427 f.; Cauville, in: BSFE 112, 1988, S. 32 (= M.D. IV, pl. 85a). S. auch M.D. IV, pl. 64 (2. Zeile oben rechts).

${ }^{157}$ Zum Isched-Baum s. auch die parallele Aussage zum Ima-Baum in der Zeile W-33. Isched-Baum und Ima-Baum kommen zusammen in den Texten von Dendera vor, wobei Isched das Adjektiv ,herrlich (sps)“ mit sich führt, Ima das Adjektiv „einzigartig ( $\left.w^{c} t j\right)^{“}$. Vgl. dazu E. II, 25 (210) u. (211), M.D. I, pl. $28=$ RdM IV, pl. 39,14 $=\mathrm{CD}$ IX, 39,10f. Zum einzigartigen Ima-Baum s. auch: Yoyotte, in: RdE 13, 1961, 87. Der ,herrliche (Helck 
Oh Osiris, komm zu deiner Gestalt (bpr),

Herrscher, komm zu deinem Bild ( $\left.s s^{r} m\right)$.

|34| Oh siehe, der König tut, was du wünschst.

Oh, die Priester preisen deinen Ka.

Oh Lebender, der im Osten lebt, groß ist deine Macht über deine Feinde.

Oh Sepa, $|35|$ nimm deinen Platz ein auf der $s b n$-Barke ${ }^{158}$, Der-dessen-Nase-lebt ${ }^{e}$ komm, sieh dieses.

Oh Schwacher ( $\underline{b} s t j)$, sei nicht schwach $(\underline{b} s t j)$ an deinem Herzen.
Oh Wenennefer, komm zu deinem Leib (d.t.t),

Merti, komm zu deinen beiden Frauen (rpw.tj=Isis u. Nephthys).

Oh siehe, der Herrscher |35| führt für dich das Ritual (š́m-bnns) durch.

Oh, die Gottesväter rezitieren für dich die Rituale.

Oh Hetep, der sich niederläßt in Manu (unterägyptische Seite des Raums = Westen), es erwachen für ihn die, die in ihren Schreinen schlafen.

Oh Sokar, |36| der dauert in der Henu-Barke, du bist vollständig an deinem Leib im Per-Nub.

Oh Herzensmatter, sei nicht matt an deinem Herzen.

Die Hekenu vertreiben (?) für dich durch Hymnen (Hekenu) (oder: du hast vertrieben (?) die Hekenu durch Hymnen ?).

übersetzt ,heilige") Isched-Baum“ spielt hauptsächlich eine große Rolle bei der Darstellung "Aufschreiben des Königsnamens auf dem Isched-Baum“. Eine Vielzahl von Belegen findet man bei Helck, in: ZÄS 82, 1957, S. 117 ff. Zusammen mit dem „einzigartigen Ima-Baum“ erscheint der „herrliche Isched-Baum" im Raum „L“ von Edfu, wobei der „einzigartige Ima-Baum“ zweimal (symmetrisch) auf der Westwand dargestellt ist, der „herrliche Isched-Baum" auf der Nord- und Südwand und außerdem auf der Ostwand zweimal die „große Akazie“ (vgl. E. IX, pl. XXIX a b).

Man kommt nicht umhin zu fragen, ob sich in unserem Text die Nennung der beiden Bäume, mit der gleichzeitigen Nennung von Begriffen des Westens (Platz nehmen) und des Ostens (sich entfernen - hier verneint) nicht auf die bekannte Deckendarstellung der Wabet (z.B. C.D. IV, pl. 315) bezieht, wo der Hathortempel bei der Himmelsdarstellung von zwei Bäumen flankiert ist. Der Baum, der dabei von der neugeborenen Sonne bestrahlt wird, wäre dann der des Ostens, entsprechend der unbestrahlte der Baum des Westens. In der Beischrift zu dieser Szene (s. C.D. IV, S. 271) ist auch richtig der westliche der Bäume als Isched-Baum bezeichnet, während der andere keine Beischrift hat. Daumas, in: ASAE 51, 1951, S. 375, bezieht die eine Beischrift auf beide Bäume. Bei seiner Beschreibung der Szene verweist er (S. 379) außerdem u.a. auf eine Textstelle E. I, 569 (=A1liot, Culte d'Horus, S. 398), wo Priester beim Neujahrsfest Garben des Isched-Baumes tragen. Es ist für unsere Überlegung wichtig, daß diese Priester Garben von Ima-Bäumen und von Isched-Bäumen tragen. (Alliot, a.a.O.: „... portant des

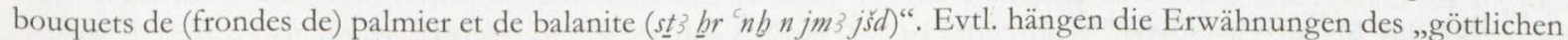
Morgen“ bzw. des „, $s^{3}-t_{5}$ “ unmittelbar vor der Nennung der Bäume mit der Aufteilung in Osten und Westen zusammen. Eine grundsätzliche Aufspaltung des Textes in eine Ost- und eine Westhälfte über den Anbringungsort hinaus ist $\mathrm{m}$. E. aber nicht gegeben, man vergleiche etwa die Zeilen O-34 und W-35. Siehe auch die Überlegungen unten in Anm. 179 (Sepa - Sokar).

${ }^{158}$ Wortspiel zwischen zbn-s.t (Platz einnehmen) und sbn-Barke. 
Oh Orion ${ }^{159}$, nähere dich den beiden Him$\operatorname{meln}^{160}$.

Oh Naref, du bist geschützt, $|36|$ nicht wirst du bedrängt ( $n$ 3r-k), du hast Macht über die Feinde gegen dich.

Oh Chenti-Imentet ${ }^{161}$, du wirst erblickt in deiner Gestalt, Djebati, du bist bekleidet mit deinen ...

Oh Unversehrt-Erwachter, komm in Frieden, |37| du mögest erblicken deine Götterneunheit bei deinem Vorbeiziehen.

Oh Körperlich-Wiedervereinter ${ }^{162}$, komm in einem Mal. Es gibt keine Widersacher deines Thrones.

Oh Merti ${ }^{163}$, komm zu deinem Opfer, mögest du dich setzen in Muße nach |38| deinem Belieben $^{164}$.

Oh Frischmacher (?) des Lebens $\left(w^{3} \underline{d} n n^{c} n b\right)^{165}$ für die Götter und Menschen, komm, nicht gibt es ein Abweisen deiner Angelegenheit.

Oh Erster des Westens, göttlicher Gott in Dendera, Herrscher, der in Ewigkeit lebt.

Oh |39| Erster des Palastes, der rein ist von Übel, die Luft trete ein in deine Nase, Osiris, Erster des Westens, großer Gott, Herr von Abydos, Sokar-Osiris, $|40|$ großer Gott in Dendera.
|37| Oh Schwarzer $(k m j j)^{166}$, der vereinigt ist (? jb? ? mit ... einzig, so weit die Götter sind $(r \mathrm{~km} n \underline{\mathrm{trw}} \text { ? })^{167}$.

Oh Oberägyptischer Upuaut, erhebe dich $(2 \times)$, es gibt für dich Jauchzen täglich.

Oh Chenti-Mekes ${ }^{168}$, deine Feste dauern, |38| man huldigt dir im Land ...

... dein Platz ist dein Lieblingsplatz ${ }^{169}$.

Oh $w^{c}-m$-šnd.t (Einziger in der Akazie ?) ${ }^{170}$, der König (nt $r-n f r)$ und die Fekti-Priester machen für dich Lobpreis.

|39| Oh ...[tp? ] ...

... du hörst Lobpreis in Ägypten $(j d w b-h r r)$, Osiris,

Herr von Busiris, großer Gott, Herrscher der Ewigkeit, Sokar-Osiris, göttlicher Gott in butt-ntr (Dendera?).

${ }^{159}$ Als Orion wird Osiris in MD IV, 66, oben Mitte bezeichnet, als Ba des Osiris in Brugsch, Thes., S. 9. Die Wortspielbeziehung zwischen Orion $(s 3 b)$ und dem Verbum „sich nähern $(s 3 h)^{\prime}$ ist naheliegend und schon in den Sargtexten bezeugt (CT III, 263a, s. LÄ, s.v. Orion; für die Spätzeit, s. z. B. Brugsch, Thes., S. 85 f.).

${ }^{160}$ Zur Formulierung ,sich den beiden Himmeln nähern“" s. E. I, 17,20 (Blackman/Fairman, in: Misc. Gregoriana, S. 408 (Z. 8).

${ }^{161}$ Herr der Dat. Hier im Gegensatz zu Chenti-Mekes ?

${ }^{162}$ Man hat den Eindruck, daß dieser Osirisbeinamen eine Beziehung zum vorangehenden „Unversehrt-Erwachten“ hat. Vielleicht hätte dieser Vers eigentlich auf die gegenüberliegende Wand gehört, so daß der nachfolgende, „Oh Merti ..." anfangende Vers, bei dem es heißt, daß er „sich setzen“ solle „in Muße“, gegenüber von dem Fragment zu stehen gekommen wäre, bei dem es ebenfalls um das Sitzen geht ( „... dein Platz ist dein Lieblingsplatz“).

${ }^{163}$ Dieser Beiname erscheint häufiger in Verbindung mit Opferhandlungen.

${ }^{164}$ S. Anm. 162.

${ }^{165}$ Beiname $\mathrm{m}$. W. sonst nicht belegt.

${ }^{166}$ Das Wort ist hier nicht mit den Haaren determiniert, wie es im WB V, 130,8 belegt ist. Die spezielle Bedeutung dieses Osirisbeiwortes ist unbekannt. Da West- und Ostwand bei unserem Text häufig in Opposition zueinander stehen, könnte man vermuten, daß „Schwarzer“ ein Gegensatz zu „Orion“ ist. Wenn nun Orion die himmliche Sphäre bezeichnet, so könnte „,kmij" die irdische Sphäre meinen, evtl. aus dem Zusammenhang „km.t-Schwarzes Land = Ägypten“. Evtl. muß man aber , ,kmjj" auch von „,km = vollständig machen, vollenden“ ableiten. 
Neige dein Angesicht gütig für den König von Ober- u. Unterägypten, den Herrn der beiden Länder (leere Kartusche), den Sohn des Re, Herr der Diademe (leere Kartusche).

Er ist der Falke, der auf dem Serech dauert, der Fürst der Untertanen, ewig.
|40| Neige dein Angesicht gütig für ...

Er ist der Falke, der auf dem Thron dauert, der Fürst der Untertanen, ewig.

\section{Gliederung:}

Die beiden Texthälften sind weitgehend parallel aufgebaut, wobei in den jeweils ersten 28 Zeilen zwischen einem Textteil mit oberägyptischem Bezug auf der Ostwand und einem Textteil mit unterägyptischem Bezug auf der Westwand unterschieden wird. Beide Textteile haben dabei eine eigene Überschrift: „Osiris anbeten, seinen $\mathrm{Ka}$ in Oberägypten preisen!“ bzw. „Osiris anbeten, seinen $\mathrm{Ba}$ (?) in Unterägypten preisen“. Neben den Begriffen „Oberund Unterägypten" werden im gesamten Hymnus Begriffspaare aufgespalten und auf die beiden Wände verteilt, ohne daß sich dabei eine innere Beziehung zu einer der beiden Landeshälften oder zu der Hälfte eines anderen Begriffspaares ergeben muß. Wenn also der $\mathrm{Ba}$ in der Einleitung der Hymne mit Unterägypten, der Ka aber mit Oberägypten verbunden wird, so darf man daraus nicht schließen, daß der Ba mit Unterägypten mehr zu tun hat als der $\mathrm{Ka}^{171}$.

An die beiden Überschriften angehängt, folgen die geographisch relevanten Aussagen des Textes. Mit einer Ausnahme (Z.O-15) stets durch die Formel ,jn jw- $k^{* 172}$ eingeleitet, werden 20 oberägyptische Gaue und 20 unterägyptische Gaue genannt. Für Oberägypten kommt diese Zahl dadurch zustande, daß man von den traditionellen 22 Gauen den typhonischen 19. o.äg. Gau unterdrückt und statt des 17. o.äg. Gaues das typhonische Gebiet von Saka ${ }^{173}$ an den Text des 16. o.̈̈g. Gaues anhängt, es also nicht selbst zählt. Eine solche Reduzierung der ägyptischen Gaue auf $2 \times 20$ Gaue ist wohl aus Gründen der Symmetrie erfolgt. Zählt

\footnotetext{
${ }^{167}$ Das ist wohl ein Wortspiel zwischen "kmjj" und „kem".

${ }^{168}$ Herr der beiden Länder (s. Ca u ville, La théologie d’Osiris à Edfou (BdE 91), 1983, S. 53), hier im Gegensatz zu Chenti-Imentet?

169 S. Anm. 162.

${ }^{170}$ Beiname $\mathrm{m}$. W. sonst nicht belegt.

171 Zum Problem des Dualismus s. Otto, in: AnOr 17, 1938, S. $10 \mathrm{ff}$.

${ }^{172}$ Die Formel ,jn jw-k“ wird gerade im Zusammenhang mit unserem Text sehr unterschiedlich übersetzt, obwohl man sich weitgehend einig ist, daß es sich hier um eine rhetorische Frage handelt, s. dazu die Rezension von P. Vernus zu „David Silverman, Interrogative Constructions with $J n$ and $J n$ - $J w \ldots 1980^{c}$, in: CdE 57, 1982, S. 248, mit weiteren Zitaten aus griech. röm. Zeit. Das Problem ist in unserem Text hauptsächlich, wie weit sich die Frage im Satz erstreckt. M. E. ist der in (rhetorische) Frage gestellte Teil des Satzes als sehr kurz anzusehen. Er beschränkt sich auf die in jedem Abschnitt variierte Ortsangabe, erstreckt sich also keinesfalls auf Angaben, die den Schutz des Osiris in jedem Ort betreffen. Der rhetorischen Frage steht eine Antwort, eine positive Aussage gegenüber. Dies muß auch im Text B schon so sein, wo die Passagen wesentlich kürzer sind als die des Textes A, z. B. „Bist du in Abydos, der Stadt des Cheper ? - so lebst du in Areq-heh." Würde man das "jn" fortlassen, hätte man einen leicht verständlichen Satz , ${ }^{*}$ Du bist in Abydos, der Stadt des Cheper, indem du lebst in Areq-heh." Die Voranstellung des „jn“ vor "jw" bewirkt also offensichtlich nur die Infragestellung der ersten Satzhälfte, während die zweite Satzhälfte davon nicht betroffen ist. Zu , jn-jw-k" als rhetorische Frage s. auch Daumas, Mamm. 431,2.

${ }^{173}$ S. L $\ddot{A}$, s. v. Saka.
} 
man die beiden abschließenden Anrufungen für Ober- und Unterägypten der Zeilen 28 hinzu, so erhält man die klassische Zahl $42^{174}$. Sehr schwierig zu beantworten ist die Frage, wer eigentlich in der 2. Sing. angeredet ist. Sicher ist es in irgendeiner Form Osiris, aber dadurch würde sich noch nicht die potentiell gleichzeitige Gegenwart des Gottes an den verschiedenen Orten erklären, die sich aus der Art der Fragen ergibt. Der Ba des Osiris scheint hier in seiner Eigenschaft angesprochen zu sein, zur gleichen Zeit ungeteilt z. B. in Elephantine und in Behedet zu sein. M.E. könnten zwei Erscheinungsformen des Osiris diesem Anspruch genügen, der Nil und der Mond. Beide können gleichzeitig an allen diesen Orten sein. Gefühlsmäßig würde ich meinen, daß der Hauptaspekt, der hier angesprochen ist, der des Mondes ist, denn der Mond wird im Osiriszusammenhang in Dendera stark betont ${ }^{175}$.

Die Zeile 28 ist einer der Pfeiler des gesamten Textes. Schon die Feststellung, daß die Aussage der Zeile bei beiden Texthälften mit dem Zeilenanfang beginnt und dem Zeilenende aufhört, verleiht ihr ein besonderes Gewicht gegenüber den anderen Zeilen ${ }^{176}$. Weiteres Gewicht erhält die Zeile durch die strenge Symmetrie zwischen der oberägyptischen und der unterägyptischen Seite. So entsprechen sich:

\begin{tabular}{|c|c|}
\hline Z. O-28 & Z. W-28 \\
\hline $\begin{array}{l}\text { Städte }(n j w . t) \\
\text { Gaue }(s p 3 . t) \text { der Götter } \\
\text { Kultbild }\left(s s^{\prime} m\right) \\
\text { Heiligtümer }(b m w) \\
\text { Machtwesen }(s b m) \\
\text { Abbild }(s b m) \\
\text { leben }(n b) \\
\text { Ewigkeit }(n h b ̣)\end{array}$ & $\begin{array}{l}\text { Stätten }(j 3 . t) \\
\text { Heiligtümer }(j t r . t j) \text { der Götter } \\
\text { Bild }(t w t) \\
\text { Heiligtümer }(g s-p r) \\
\text { Götterneunheit }(p s \underline{d} . t-n \underline{t} r w) \\
\text { Bild ( } \underline{b} n t j) \\
\text { gesund sein }(s n b) \\
\text { Ewigkeit }(\underline{d} . t)\end{array}$ \\
\hline
\end{tabular}

Es ist klar, daß auch die Worte, die zwischen den oben aufgezählten, den Kontext prägenden Wortpaaren stehen, sich in irgendeiner Weise entsprechen ${ }^{177}$.

Mit der Zeile 29 fängt in beiden Texthälften ein neuer Abschnitt an. Dabei stellt die Nennung der Himmelsrichtungen die Verbindung zu der vorangehenden Texteinheit dar: Die

\footnotetext{
${ }^{174}$ Die Zahl der Gaue. Wie schon angemerkt, wird bei einem Gau (Z. O-15) die Formel „jn-jw-k" nicht benutzt.

${ }^{175}$ Man denke an: Derchain, „La pêche de l' œil es les mystères d'Osiris à Dendara“, in: RdE 15,1963, S. $11 \mathrm{ff}$. Es ist in diesem Zusammenhang interessant, daß der Mondaspekt des Osiris gerade in Verbindung mit der Übertragung seiner Herrschaft an seinen Sohn unterstrichen wird, etwa (MD IV, 77 unten) „... er (Osiris) fliegt auf zum Horizont als Mond, er erleuchtet die beiden Länder wie der Horizontische im Horizont. Er übergibt das große Amt seinem Sohn, den er liebt, dem König von Ober- und Unterägypten, dem Herrn der beiden Länder (leere Kartusche) auf seinem Thron in alle Ewigkeit“. Interessant auch die Bezeichnung des Osiris als „heißer Stier (= Mond)" in MD IV, 66 oben rechts. Zur Beziehung Osiris-Mond allgemein s. Derchain, in: La lune (Sources Orientales 6) 1962, S. 44 ff., Herbin, in: BIFAO 82, 1982, S. 237 ff. bes. S. $261 \mathrm{f}$. u. Cauville, in: BSFE 112, 1988, S. 34f. (Anm. 27 = Dümichen, GI I, pl. 100 ?).

${ }^{176}$ Diese Feinheit kann man allerdings bei der Abschrift von J. Dümichen entdecken, da bei seiner Textwiedergabe der Zeilenumbruch O-27/28 und O-28/29 falsch wiedergegeben ist. Man hat den Eindruck, daß die Zeilen 28 vom für das Layout Verantwortlichen zuerst festgelegt wurden.

177 S. o. S. 15
} 
Nennung von Süden und Osten schließt dabei richtig an die Aufzählung der Gaue Oberägyptens an, die Nennung von Norden und Westen an die der Gaue Unterägyptens. Damit ist ein kurzer Abschnitt eingeleitet, den man vielleicht mit „du bist in allen Himmelsrichtungen, Osiris“ thematisieren kann, wobei neben unseren vier Himmelsrichtungen auch Himmel und Unterwelt eine Rolle spielen. Möglicherweise spielt in dem nachfolgenden, an den Himmel anknüpfenden Gedankengang, daß die Sterne und besonders Sothis im Gefolge des Gottes sind, auch die Vorstellung vom Orion als einer Ba-Vorstellung des Osiris eine Rolle. An die Nennung der Sothis knüpft dann folgerichtig eine Passage über das fruchtbare Überschwemmungswasser an, das das abschließend genannte Opfer erst ermöglicht. Die entsprechenden Sätze auf der Westwand sind leider stark zerstört. Entscheidend ist hier wohl - als Opposition zu Himmel - die Nennung der Dat. Die nicht lesbare Gruppe, die im Gefolge des Gottes ist, dürfte wohl, im Gegensatz zu den Sternen, mehr erdgebunden sein. Auch die nachfolgende Nennung der Berge mit den „göttlichen Gesteinen“ hängt wohl vom Begriff der „Dat“ ab. Im einzelnen entsprechen sich in den Zeilen 29:

\begin{tabular}{|c|c|}
\hline Z. O-29 & Z. W-29 \\
\hline $\begin{array}{l}\text { Süden } \\
\text { Hoheit }(s f j . t) \\
\text { Osten } \\
\text { Respekt }(n r w) \\
\text { Himmel } \\
\text { Ba }\left(b_{3}^{2}\right) \\
\text { Sterne } \\
\text { Brot, Fleisch, Wein }\end{array}$ & $\begin{array}{l}\text { Norden } \\
\text { Schrecken }(b r j . t) \\
\text { Westen } \\
\text { Zittern }\left(s d^{3} d^{j}\right) \\
\text { Dat } \\
\text { Körper }(t j . t) \\
\text {...-Figuren } \\
\text { göttliche Gesteine }\end{array}$ \\
\hline
\end{tabular}

Dieser Abschnitt wird abgelöst durch die Vorstellung der Gottheit und der Berechtigung ihrer Ansprüche. Als Zeichen dieser Vorstellung wird dabei einleitend das emphatische „du bist ja (twt r-k bzw. ntk)" (Z. O-30 und Z. W-31) verwendet. Der zweite Teil dieses Abschnittes preist das Aussehen der Gottheit, er wird eingeleitet durch „wie schön ist ( $n-w j$ bzw. $n f r-w j$ ) (Z. O-31 und Z. W-32).

\begin{tabular}{|c|c|}
\hline Z. O-30f. & Z.W-31f. \\
\hline $\begin{array}{l}\text { Fürst der Götter (sr ntrw) } \\
\text { ältester Sohn des Iri-pat (Geb) } \\
\text { Verpflichtungen ( } n t-\text { - }) \\
\text { ewig }(n h \underline{h}) \\
\text { Sonnen-(?)-scheibe } \\
\text { vergehen }(m r h)\end{array}$ & $\begin{array}{l}\text { Herrscher der Tempel ( } \not k, \text { bw.wt }(n \underline{t} r . w)) \\
\text { goldenes Abbild (Sohn) der Nut } \\
\text { Bestimmungen (jrw) } \\
\text { ewig ( } . . t) \\
\text { Mond } \\
\text { mangeln }\left(w s^{r} r\right)\end{array}$ \\
\hline
\end{tabular}

Mit „mögest du hören die Stimme ... (,,ndb-k $m d w$ " bzw. „sd $d m(?)-k m d w$ ") (Z. O-32 und Z.W-33) beginnt die Einleitung zu den Anrufungen, die dann mit der Interjektion ,j" eingeleitet werden. Auch bei dieser Einleitung sind die Entsprechungen zwischen West- und Ostwand augenfällig: 


\begin{tabular}{|l|l|}
\hline Z.O-32f. & Z.W-33f. \\
\hline Stimme deines Sohnes & Stimme deiner Djerti \\
Ruheplatz & besteigen \\
Serech/Thron & Thron \\
küssen & preisen \\
„Götter, die um die Kapelle herum sind“ & Bau von Heliopolis \\
Sa-Ta & Göttlicher Morgen \\
Herrlicher Isched-Baum & Einzigartiger Ima-Baum \\
Platz nehmen & nicht entfernen \\
\hline
\end{tabular}

Weitere Gedankenpaare lassen sich durchaus noch im Text selber finden.

Der abschließende Teil wird durch die Anrufe „Oh $(j)$ “ bestimmt. In der oberägyptischen Hälfte des Textes finden wir 15 solcher Interjektionen, in der unterägyptischen Hälfte findet man dagegen nur 11, wobei man wegen der Zerstörungen der Inschrift mit einer, höchstens aber zwei weiteren Anrufungen rechnen muß. Das Ungleichgewicht bei den Anrufungen ist angesichts der sonst so stark betonten Symmetrie des Textes auffällig. Vielleicht läßt sich aber das Problem dadurch lösen, daß man annimmt, daß in einem Fall ein Begriffspaar aus Platzgründen nicht auf die beiden gegenüberliegenden Wände verteilt wurde, ich denke da an die beiden Verse, die mit „oh Unversehrt-Erwachter (O-36)“ und „oh Körperlich-Wiedervereinigter (O-37)“ beginnen. Die insgesamt wohl 28 Anrufungen (wenn man zwei zerstörte unterstellt, was aber m.E. unwahrscheinlicher ist als die Annahme nur einer zerstörten Anrufung) wären dann auf $2 \times 14$ aufgeteilt, womit die Annahme einer Beziehung zum Mond reizt. Leider ist die Westwand in diesem Bereich so zerstört, daß man bei der Rekonstruktion der Verteilung der Verse über eine Vermutung nicht hinauskommt.

Zunächst beginnt der Text mit der vierfachen Aufforderung an Osiris (in verschiedenen Namen) „komm zu ...". Es ist dabei schwer zu entscheiden, welche der danachstehenden Begriffe sich näher, welche ferner stehen: $b p r$-Bild, sšm-Bild, d.t-Leib, Frauen $(r p w . t j)$. Die vierfache Aufforderung, man möchte fast sagen „Beschwörung“ „komm“ ist auch deshalb besonders auffällig, weil dieser Imperativ, abgesehen von einem Fall in Zeile O-35, erst gegen Ende des Textes, ab Zeile O-36 auftaucht, hier ebenfalls vier Mal hintereinander, jedoch nur auf der Ostwand, die Westwand ist hier stark beschädigt.

Am Anfang bleibt die Symmetrie des Textes gewahrt:

\begin{tabular}{|l|l|}
\hline Z. O-34f. & Z.W-34ff. \\
\hline König $(n s w . t)$ & Herrscher $(j t j)$ \\
tut, was du wünschst & führt für dich das Ritual durch \\
Priester & Gottesväter \\
preisen deinen Ka & rezitieren für dich die Rituale \\
Lebender, der im Osten lebt & Hetep, der sich niederläßt in Manu (Westen) \\
Sepa & Sokar \\
Platz einnehmen & dauern \\
Sechen-Barke & Henu-Barke \\
Schwacher $(\underline{b} s t j)$ & Herzensmatter \\
sei nicht schwach an deinem Herzen & sei nicht matt an deinem Herzen \\
\hline
\end{tabular}


Mit der Aufforderung, „nicht schwach“ bzw. „nicht matt“ zu sein, endet die auffällige Parallelität der Wände zunächst, und es bleibt vor der nächsten Interjektion ein Satz übrig: „Die Hekenu (-Fremdländer ?) ${ }^{178}$ vertreiben (?) für dich durch Hekenu-Hymnen“ (?). Das Wortspiel, das in „Hekenu“ liegt, ist eindeutig. Dagegen läßt sich ein innerer Zusammenhang mit der voranstehenden Zeile nicht erkennen, besonders, weil auch in der parallelen Anordnung kein Platz für sie vorhanden wäre. Es bleibt m. E. nur der Schluß, daß die Zeile eine einleitende Funktion zu den nachfolgenden Anrufungen hat, die man ja durchaus als Hymnen ansehen kann, oder daß sie eine abschließende Funktion zu den voranstehenden Versen hat. Wenn wir aber hier eine solche Zwischenüberschrift haben, so muß mit dem Voranstehenden ein Abschluß erreicht worden sein. Da heißt es O-34 „Oh Sepa, nimm deinen Platz ein auf der shb-Barke“ und in der parallelen Zeile W-35 „Oh Sokar, der in der Henu-Barke dauert... “179 M. E. kann man den Text mit seinen 12 Anrufungen ${ }^{180}$ mit einiger Wahrscheinlichkeit auf eine Kulthandlung übertragen, die in dem Raum, in dem der Text zu finden ist, durchgeführt wurde: Die Barke mit dem Gottesbild für die Prozession ist bereit, und der König fordert den Ba oder den Ka des Gottes auf, sich darauf niederzulassen. Durch die Vereinigung mit seiner spirituellen Kraft hört der Schwache auf, schwach zu sein, und der Herzensmatte ist nicht mehr matt. Damit ist ein wichtiger Abschnitt der Prozession abgeschlossen.

Bei den nun folgenden ca. 14 bis 16 Anrufungen - die genaue Zahl ist wegen der Zerstörungen nicht sicher zu nennen, m. E. sind es nur 14+1 Anrufung - lassen sich keine sicheren parallelen Aussagen mehr feststellen. Auffällig ist, wie schon oben gesagt, die viermalige Verwendung des Imperativs „komm“ in den Zeilen O-36 bis O-38. Möglicherweise handelt es sich um den Begleittext zu einem Opfer, das jetzt vor der Prozessionsbarke durchgeführt wird, denn in der Zeile O-37f. heißt es „Oh Merti, komm zu deinem Opfer, mögest du dich setzen in Muße zu dem, was du liebst. “ Auffällig ist auch wieder die Nennung von handelnden Personen. Z. W-38: „Oh Einziger in der Akazie, der König und die Fekti-Priester machen für dich Lobpreis“"181.

Der Text endet in beiden 40. Zeilen mit der Aufforderung an Osiris, dem Herrscher gewogen zu sein, denn er sei der Falke auf dem Thron, der Herrscher der Untertanen. Mit dieser abschließenden Formulierung könnte die Verbindung zum Bandeau-Text ${ }^{182}$ hergestellt sein, wo ebenfalls der König als „bntj-k弓.w- $n b . w^{c}$ " bezeichnet wird.

\footnotetext{
${ }^{178}$ Dieser Name ist m. W. nur noch einmal in „Geographischen Inschriften“ belegt. Gauthier D.G. IV, S. 44, zitiert einen Hinweis von Drioton, daß der Name im Tempel von Médamoud vorkommt. Wahrscheinlich handelt es sich um die Stelle Médamoud Nr.169, im Zusammenhang mit dem Pehu-Gebiet von Abydos. «Car tu es le Prince de l'Iqret, éclairant l'obscurité, rajeunissant les habitants du Hekenou par ses rayons. » In diesem Text spielen anscheinend die Hekenu keine negative Rolle, die es rechtfertigen würde, daß man sie vertreibt. (Auch in TB 142,85 wird in den Handschriften Ad u. Pq ein Osiris-Ort „,hkn.t“ genannt, andere Handschriften haben allerdings „,hnk.t".) Die Rekonstruktion des Verbs muß deshalb in unserem Text sehr fraglich bleiben. Nach der Abschrift von Dümichen ließe sich vom Platz her „bsf “ ergänzen, was zu den beiden Determinativen passen würde. Vielleicht muß man als Konsequenz übersetzen: „Die Hekenu-Länder wehren für dich ab mit Hymnen“, wobei das, was abgewehrt wird, nicht genannt ist und nur die Handlung an sich wichtig ist.

${ }^{179}$ Es ist vielleicht zu spitzfindig, wenn man sich wundert, daß Sepa seinen Platz einnimmt, während Sokar schon dauert. Andererseits ist es schon auffällig, daß es in den Zeilen 33 ein ähnliches Verhältnis zu den beiden dort genannten Bäumen gibt. O-33: „Der herrliche Isched-Baum, mögest du deinen Platz einnehmen neben ihm“, W-33: „Der einzigartige Ima-Baum, mögest du dich nicht entfernen von ihm.“ Ob man nun daraus vielleicht vermuten darf, daß es sich bei den beiden Bäumen nicht um die Bäume selbst, sondern um Kultbarken aus ihrem Holz handelt, Isched-Holz für die Barke des Sepa und Ima-Holz für die Barke des Sokar, sei dahingestellt. Immerhin finden wir unter den Bahren bei MD IV, 71 (oben) den Hinweis auf Ima-Bäume.

${ }^{180} \mathrm{Ob}$ diese Zahl zufällig ist oder nicht, vermag ich nicht zu sagen.

${ }^{181}$ Zum Fekti-Priester und seiner Aufgabe bei der Wiederbelebung von Statuen s. Derchain, pSalt, S. 69-70 u. 73-75, dazu auch Vernus, in: BIFAO 76, 1976, S. 9.
} 
Die beiden Darstellungen des Textes sind wie die des Choiak-Textes in den Corpus der Inschrift eingefügt. Sie sind unbedingt Bestandteil des Textes. Beide Bilder sind gleichartig aufgebaut: Zum Tempelinneren hin finden wir jeweils eine Darstellung des Osiris auf einer Löwenbahre, wo er von zwei geflügelten Göttinnen geschützt wird. Interessanter ist die Darstellung des Königs, die sich an dieses Bild in Richtung des Tempelausganges anschließt. Der König schreitet auf diesen Ausgang zu, wendet aber den Oberkörper zu Osiris auf der Bahre um und räuchert ihm mit einem Räucherarm. Es ist dies die Darstellung, die man häufig in dieser oder ähnlicher Art bei Prozessionen findet. Der Text bestätigt, daß wir es hier mit einem Prozessionszusammenhang zu tun haben, bei dem die Zielrichtung aus dem Tempel hinaus führt. Der König „weihräuchert deinem $s \check{m} m$-Bild, Kind (und Sohn ?) der Nut. Dein Weg sei rein und ohne Übel“ (unter Z. O-17). In der entsprechenden Zeile gegenüber (unter Z. W-17) wird statt dem š̌m-Bild dem Ka geräuchert und der Gott ist der Sohn des Geb. Auch hier ist auf den Weg angespielt, den der Gott auf seiner Prozession zurücklegen muß. Durch die Reinigung mit Weihrauch ist der „Weg ... ohne Schmutz“. An anderer Stelle heißt es: „Dein Platz ist in Jubel, du bist gerechtfertigt, Sohn des Geb, du machst einen Umzug ( $p \underline{h} r$ ) um Dendera in ..." (unter Z. W-14f.). Und in der entsprechenden Darstellung der Ostseite heißt es, der König ,räuchert deinem Körper an der Spitze aller Götter, Sokar-Osiris, inmitten von ... Es kommt dein $\mathrm{Ba} \ldots(m b$ ? - zusammen mit ) deinem Ka."

Durch die wenigen Textbeispiele ist wohl deutlich, daß die Darstellung sich auf den Beginn einer Prozession aus dem Dachtempel hinaus bezieht ${ }^{183}$. Es bedarf wohl keiner gewagten Spekulation, um anzunehmen, daß es sich dabei um die gleiche Prozession handelt wie die, bei der das „Cérémonial pour faire sortir Sokaris “184 durchgeführt wird ${ }^{185}$. Es ist in diesem Zusammenhang interessant, daß in Dendera das Sokar-Zeremonial nirgends vollständig belegt ist ${ }^{186}$. Man könnte sich durchaus vorstellen, daß beim Dachtempel des Osiris in Dendera die Anrufungen unserer Hymne Teile des „Cérémonial pour faire sortir Sokaris“ ersetzen oder wenigstens begleiten, bis dann mit den Zeilen $73 \mathrm{ff}$. der eigentliche Auszug aus dem Tempel stattfand. Vielleicht wurde vorher noch jenes Ritual vollzogen, das als „Schutz der Barke“" bekannt ist und als großer Text auf der Eingangswand zwischen den genannten Zeilen des „Cérémonial pour faire sortir Sokaris" und dem oberägyptischen Teil unserer Hymne steht.

\footnotetext{
${ }^{182}$ Dümichen, GI I pl. 100.
}

${ }^{183}$ Mit dieser Auffassung bin ich anderer Meinung als S. Cauville, in: BSFE 112, Juni 1988, S. 34, die den hier besprochenen Text als Begrüßung für den aus den östlichen Osiriskapellen hier einziehenden Sokar-Osiris ansieht. Gegen Frau Cauvilles Ansicht spricht auch die Ausrichtung des Textes incl. der Anrufungen zum Tempelinneren hin. ${ }^{184}$ S. Goyon, in: RdE 20, 1968, S. 63-96.

${ }^{185}$ Der Text dieses Rituals findet sich ab der Zeile 73 (nach der Zählung von Goyon, zur Frage eines einheitlichen Ursprunges dieses Textes vgl. Goyon, a.a.O. S.67, Anm.1) an der Tür, auf die der König in unserer Szene zuschreitet. Diese Version fehlt bei Goyon (s. Goyon, a.a.O. S.64, Anm. 5), s. einstweilen: Dümichen, Baugeschichte, Taf. 32 rechts. Aus dem Bereich vor der Zeile 73 findet man in Dendera nur die wenigen Zeilen des Textanfanges, die in das Morgenlied an Osiris eingebettet sind, das sich im gleichen Raum, in dem sich unsere Hymne befindet, an den Gott im Tempelinneren wendet (s. Beinlich, in: RdE 32, 1980, S. 26ff.). Sollte es ein Zufall sein, daß gerade die Anfangszeilen des Sokar-Rituals in einem Text, der den Tagesanfang des Gottes zum Inhalt hat, verwendet werden? Könnte es nicht vielmehr so sein, daß sich das Sokar-Ritual eines Textes bediente, der ohnehin in die Handlungen des morgendlichen Aufweckens der Gottheit gehört? In jedem Fall scheint es mir beachtenswert zu sein, daß wir in dem Raum, in dem unsere Hymne steht, den Übergang von dunkel zu hell, von Nacht zu Tag durch die Anfangszeilen des Sokar-Rituals markiert finden, den Ausgang aus diesem Raum aber durch den Text, der den Auszug des Gottes in seiner Barke markiert (s. Goyon, S. 68 f.). Darf man daraus schließen, daß der ganze Text, also auch der dazwischenliegende, raumbezogen ist?

${ }^{186}$ Vgl. dazu Goyon, a.a.O. S. 69, Anm.1. 


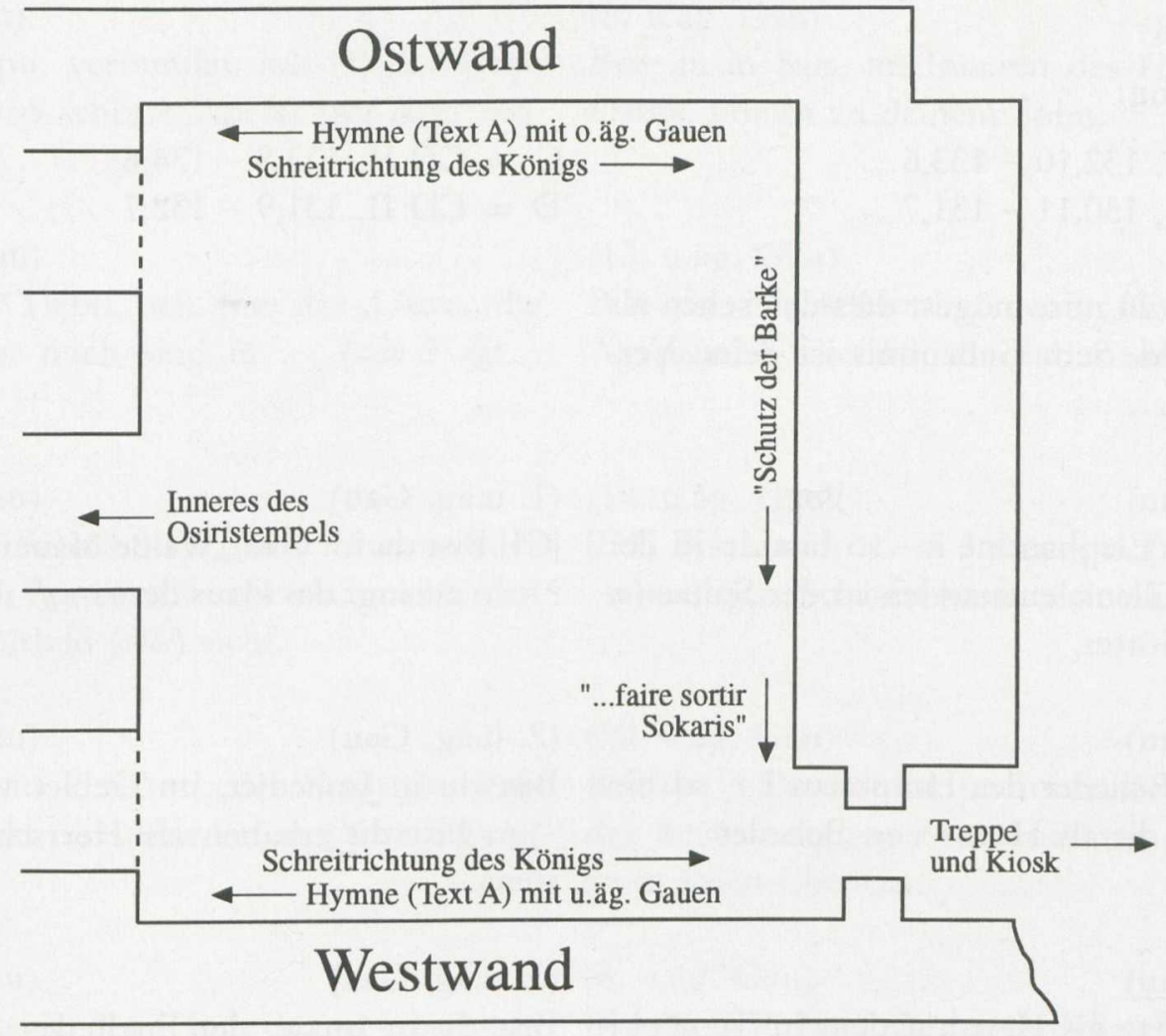

Abb. 1. Skizze vom 1. Raum der westlichen Osirisräume auf dem Dach des Dendera-Tempels

Text B ( Dendara II, S. 130-134)

Publikation: Auguste Mariette: Dendérah, Tome II, Planches, Paris 1870, pl. 39.

Johannes D ümichen: Geographische Inschriften altägyptischer Denkmäler, Zweite Abtheilung - Tafeln (= Brugsch: Recueil de monuments égyptiens, Bd. IV), Leipzig 1866, Taf. 27-29. Emile Chassinat: Le temple de Dendara, Tome II, Le Caire 1934, S. 130-134.

Es war schon angedeutet worden, daß die einzelnen Teile des Textes A sich z. T. erheblich voneinander unterscheiden. D.h. man muß damit rechnen, daß sich die Hymne aus Teilen unterschiedlicher Herkunft zusammensetzt. Ein Hinweis darauf könnte sein, daß es zu dem ersten, dem geographischen Teil der Hymne eine Parallele gibt ${ }^{187}$, hier als Text B bezeichnet, die aber durchaus ihren eigenen Charakter hat und weit davon entfernt ist, nur eine verkürzte Version des Textes zu $\operatorname{sein}^{188}$.

${ }^{187}$ Text B $=$ CD II, S. 130-134; die Reihenfolge der Zeilen ist übersichtlicher bei Mariette, Dendérah II, pl. 39 und Dümichen, GI II, pl. 27-29.

${ }^{188}$ Ich verzichte hier auf eine Kommentierung der fortlaufenden Übersetzung, da alle Teile entweder bei der Übersetzung des Textes A weiter oben berücksichtigt wurden, oder - Einleitung und Schluß - im folgenden kommentiert werden. 


\section{Übersetzung:}

Zeilenzählung:
$\mathrm{A}=\mathrm{CD}$ II, $132,10-133,6$
$\mathrm{C}=\mathrm{CD}$ II, $133,8-134,6$
$\mathrm{B}=\mathrm{CD}$ II, 130,11 - 131,7
$\mathrm{D}=\mathrm{CD}$ II, $131,9-132,7$

|A1| Komm zu mir, mögest du mich sehen als dein $s s ̌ m$-Bild. Sein Geheimnis ist deine Verborgenheit ...

(1. o.äg. Gau)

Bist (du in) Elephantine ? - so bist du in der Stadt des Füllens, entstanden an der Spitze ( $m$ b̧ว.t) aller Götter.

\section{(2. o.äg. Gau)}

Bist du in Behedet des Horussees ? - so bin (ich) neben dir als Horus von Behedet.

\section{(3. o.äg. Gau)}

Bist du in Fag als Herr auf dem ...-Tempel ? - |A2| so hat der Tempel des rechten Auges deine Schönheit.

\section{(4. o.äg. Gau)}

Bist du in Theben, der Herrin der göttlichen Gaue Ägyptens? - so hast du dort du dein Entstehen begonnen.

\section{(5. o.äg. Gau)}

Bist du in Koptos im Haus ... ? - so schützt Senut deinen Leib.

\section{(6. o.äg. Gau)}

Bit du in Iunet im Tempel des Sema-taui ? so bin ich neben dir als Harsomtus.

\section{(7. o.äg. Gau)}

Bist du in Kenemet des Sistrum-Hauses ? - so macht das Benu-Haus, was du begehrst.

\section{(8. o.äg. Gau)}

Bist du in Thinis, $|\mathrm{A} 3|$ der Stadt des Cheper? - so lebst du in Areq-heh.
(1. u.äg. Gau)

|C1| Bist du im Gau „Weiße Mauer" in $t p h . t-n n$ $?$ - so erzeugt das Haus des $r s-w \underline{d} ?$ deine Dinge.

(2. u.äg. Gau)

Bist du in Imhedjet, im Gebiet von Chem ? - so bist du erhaben als Herrscher des Westens.

\section{(3. u.äg. Gau)}

Bist du in Imaw, der Stadt des Apis ? - so schützt der Tempel der Sechat-Hor deinen Körper.

\section{(10. u.äg. Gau)}

Bist du in Athribis ... |C2| in Hap-iutiu ? - so siehst du die Gestalt deines Sohnes (*so siehst du, was dein Sohn tut).

(9. u.äg. Gau)

Bist du in Busiris, im Gebiet des Preisens? so ist Anedjti überschwemmt mit deinem jr.w-Bild.

\section{(4. u.äg. Gau)}

Bist du im 4. u.äg. Gau, in Djeqa-per? - so hast du dich niedergelassen in $\not h-w r$.

\section{(7. u.äg. Gau)}

Bist du in Senetj-nefer im 7. u.äg. Gau ? - so hat Hut-Nehet deinen Respekt ...

\section{(6. u.äg. Gau)}

|C3| Bist du in Chasut, dem Haus des Königtums des Re? - so ist das Verborgene darin der Schutz deines Leibes. 
(9. o.äg. Gau)

Bist du in Ipu, verbunden mit (?) der Doppelmaat ? - so schützt Aperet-Iset dein š́mBild.

\section{(10. o.äg. Gau)}

Ich bin in *'Tjebti, ich bin der Löwe, die Feinde gegen mich sind in ... (ndn ? ntw Fessel ?).

\section{(11. o.äg. Gau)}

Bist du in Schashotep (im) Inneren des Hut-nememet ? - so wünscht man nicht, daß man dein Kultbild (š̌m) sieht.

(12. o.äg. Gau)

|B1| Bist du in Per-Anti, der Stadt des Falken ? - so bist du zufrieden in „Vereinigung der Freude".

\section{(13. o.äg. Gau)}

Bist du in Assiut, dem Haus deines Gottesleibes ? - so ist dein Mysterium verborgen am Platz des Vereinigens.

\section{(14. o.äg. Gau)}

Bist du in Kusae, deiner göttlichen Kapelle ? - so ist der Schutz deines Ausflusses (Kebehsen)uef.

\section{(15. o.äg. Gau)}

Bist du in Hermopolis, dem ersten Gau, in dem das Licht angefangen hat? - |B2| so hat der Tempel der Maat deine Vollkommenheit (nfrw).

\section{(16. o.äg. Gau)}

Bist du in Hebenu im Haus des Triumphes $\left(m_{3}{ }^{c}-b r w\right)$ ? - so bist du zufrieden als Herr der Herzensweite.

\section{(18. o.äg. Gau)}

Bist du in Anti (Dunanui ?) im Inneren der Gotteshalle ? - so siehst du auf mich als Iunmutef. Ich bin der Fekti-Priester, der seinen Vater täglich erhaben macht, ohne daß ich müde werde, seine Feinde zu schlagen. Oxyrhinchos
(5. u.äg. Gau)

Bist du in Sais, im Inneren des Hut-Biti ? Osiris, komm zu deinem Sohn.

\section{(13. u.äg. Gau)}

Bist du in Heliopolis, dem „Himmel von Ägypten"? - so läßt du dich nieder...

(18. u.äg. Gau)

|D1| Bist du in ... in Us-em-pet (ws-m-p.t) ? - so schützt Scha-en-bastet deine Dinge.

\section{(20. u.äg. Gau)}

Bist du in Per-Sopdu, im Inneren des Hauses der Rechtfertigung ? - so nimmst du Gestalt an in Imen-Cheperu.

(8. u.äg. Gau)

Bist du in Pithom, an der östlichen Mündung? - so (leb)st du als (Leb)ender (Gott) ...

(19. u.äg. Gau)

(Bist du in ...), |D2| dem Platz Sched-suwadjet? - so jubelst du in der Wüste des Jubelns.

\section{(14. u.äg. Gau)}

Bist du in Tjaru, an den Horuswegen ? - so ... ersinnen gegen deine Majestät.

\section{(15. u.äg. Gau)}

Bist du in der Thot-Stadt, im Hut-meriti ? so bist du dauerhaft im Per-wenech.

\section{(16. u.äg. Gau)}

Bist du in Mendes, im Haus der Götterneunheit ? - |D3| so jauchzt deine Majestät über die Tätigkeit (kว.t) der Frauen. 
ist niedergeworfen, seine Bewohner sind im Feuer, $|\mathrm{B} 3|$ die Habgierigen (die Einwohner der Stadt) sind geschlachtet in der Glut.

(20. o.äg. Gau)

Bist du in Herakleopolis, in den beiden südlichen Heiligtümern? - so ist deinem Kopf die große Atef-Krone aufgesetzt.

(21. o.äg. Gau)

Bist du in Tehenet $\left(\underline{t} h n-n-\check{s}^{c}-\underline{b n} n\right)$ ? - so bist du geformt in Neb-irt.

(22. o.äg. Gau)

Bist du in Tep-ihet, im Inneren des Perwer? - so schützt Hathor deine Glieder. |B4| Der 22. o.äg. Gau sticht, um niederzuwerfen den ... (Seth), um zufrieden zu machen das Herz des Osiris ...

Komm auf mein Wort, mein Vater Osiris. Ich bin dein Sohn Horus, gerechtfertigt. Osiris Wenennefer, gerechtfertigt, großer Gott inmitten von Dendera, neige dein Angesicht gütig für den König von Ober- und Unterägypten, den Herrn der beiden Länder (leere Kartusche).
(12. u.äg. Gau)

Bist du in Tjeb-netjeret, dem Tempel des Heri-ideb (Schu)? - so sind deine Feinde getötet in Hut-(biti ?)-Hor.

(17. u.äg. Gau)

Bist du in Sema-behedet, dem Platz des Vereinigens der beiden Länder? - so ist

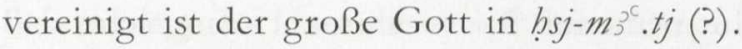

Du bist der Lebende, durch dessen Anblick man lebt, alle Menschen leben von dir. |D4| Du bist der Herr des Windhauches, den er dem gibt, der abwendet alles (? twt-s ?) von dem, der deinen Namen nennt (dm-rn-k). Ganz Ägypten führt für dich herbei seine Dinge. Du bist der Herr von ihnen allen. Du bist Osiris, machtvoll an seinem Leib, der entstanden ist aus (als ?) Amun-wer.

Der große Unterschied zu der langen Hymne liegt - abgesehen vom Umfang - darin, daß hier in Text B der Sprecher auch innerhalb des Textes eine aktive Rolle hat. Es ist dies, wie die Anfangsworte zeigen, Horus, der zu seinem Vater Osiris spricht. Er fordert Osiris auf: „Komm zu mir, mögest du mich sehen als dein š̌m-Bild. Sein Geheimnis ist deine Verborgenheit". Natürlich ist Horus eigentlich der regierende König, wie auch am Schluß des Textes gesagt wird. Das Verhältnis zwischen Osiris und Horus/König ist durch das Wort „š́m-Bild“ bezeichnet. Nach Ockinga ${ }^{189}$ ist „,šm.w als eine Bezeichnung für den König ... nur verhältnismäßig selten belegt“. Seine weitere Interpretation, „der König sei das orakelerteilende Bild des Gottes“ ${ }^{\text {190 }}$, läßt sich aus unserer Textstelle nicht bestätigen. M. E. ist die Interpretation von dem „orakelerteilenden Bild“ etwas zu weit gegriffen. Ich meine, daß man an ein durch das Einwohnen des Ba aktiviertes Bild denken sollte. Der König wäre dann auf Grund der ihm innewohnenden göttlichen Kraft evtl. auch zur Orakelerteilung fähig. In unserem Text müßte man die Stelle so interpretieren, daß Horus/König den Ba des Osiris auffordert, in ihn

\footnotetext{
${ }^{189}$ Boyo Ockinga, Die Gottebenbildlichkeit im Alten Ägypten und im Alten Tesrament (Ä ÄT 7), 1984, S. 45. ${ }^{190}$ A. a. O., S. 49.
} 
einzuwohnen, etwa in dem Sinne: ,Komm zu mir, Ba des Osiris, mögest du mich als dein $s \check{s} m$-Bild amsehen und in mir Platz nehmen. Das Geheimnis (hier vielleicht wirklich „Mysterium") dieser $55 m$-Verbindung zwischen dir und mir ist, daß du darin, d.h. in mir verborgen bist $^{6191}$.

Text B behält die Sprecherrolle des Horus aus der Einleitung im Text bei. In allen Gauen, in denen ein Falkengott der Hauptgott dieses Gaues ist und den Schutz des Osiris in dem Gau gewährleistet, identifiziert sich Horus/König mit dieser speziellen Form und spricht in der 1. Sing. von sich. Im 2. o.äg. Gau ist er Horus von Behedet, im 6. o.äg. Gau Harsomtus, im 10. o.äg. Gau ist er der Löwe und im 18. o.äg. Gau identifiziert sich Horus/König mit Iunmutef, der für seinen Vater in der Rolle des Fekti-Priesters handelt ${ }^{192}$. Im unterägyptischen Teil wird zwar die 1. Sing. nicht verwendet, aber immerhin wird zweimal die Formulierung „dein Sohn“ verwendet (im Zusammenhang mit Athribis und mit Sais), eine Ausdrucksweise, die auch im Abschluß des oberägyptischen Teils zu finden ist. Horus/König legitimiert sich vor Osiris mit den Worten „Ich bin dein Sohn Horus, gerechtfertigt.“

Auffällig am Text B ist auch die Reihenfolge der unterägyptischen Gaue, die weder mit der des Textes A, noch mit der einer anderen Gauaufzählung übereinstimmt ${ }^{193}$. Die Reihenfolge der unterägyptischen Gaue sieht folgendermaßen aus: 1, 2, 3, 10, 9, 4, 7, 6, 5, 13, 18, 20, 8, $19,14,15,16,12,17^{194}$. Diese Reihenfolge der unterägyptischen Gaue im Text B ist aber keineswegs willkürlich. Sie zählt zunächst die westlichen Deltagaue auf, wobei sie von Memphis ausgeht. In einem 2. Schritt werden dann, mit Heliopolis beginnend, die östlichen Deltagaue genannt. Da der 17. u.äg. Gau als äußerster Norden gilt, schließt mit ihm die Aufzählung.
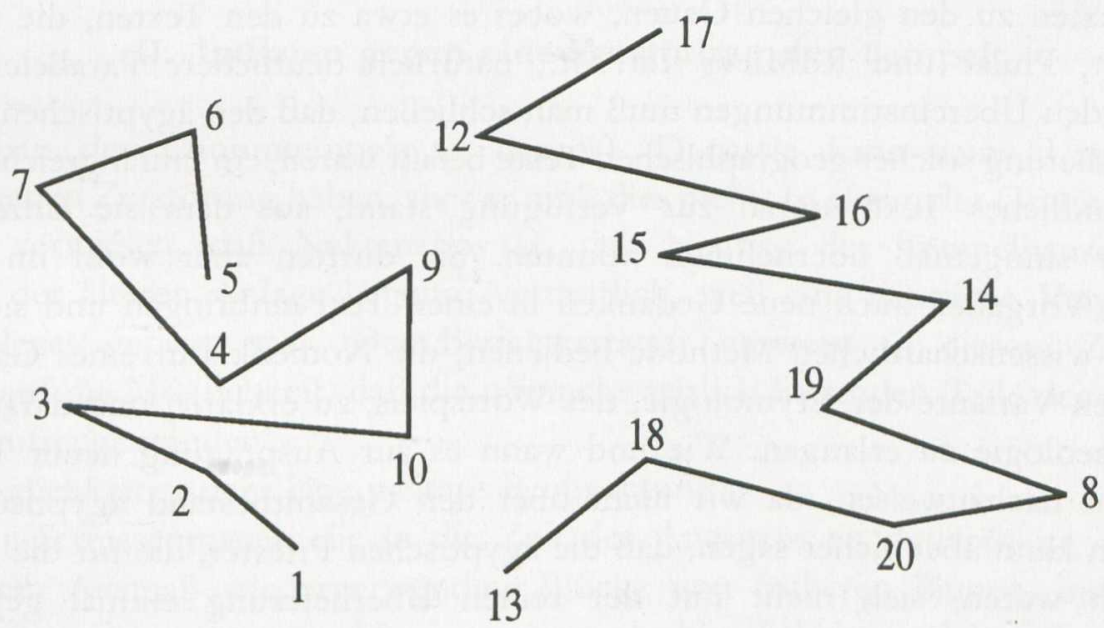

Abb. 2. Schema der Reihenfolge der unterägyptischen Gaue im Nildelta nach Text B

\footnotetext{
191 Zur Verborgenheit des š̌m-Bildes vgl. Ockinga, a.a.O., S. $41 \mathrm{ff}$.

${ }_{192}$ Erstaunlicherweise spielen diese beiden Bezeichnungen im pJumilhac keine Rolle ! Zum Fekti-Priester s. oben, S. 23.

${ }^{193}$ Vgl. etwa Helck, Gaue, S. 133.

${ }^{194} \mathrm{Jm}$ Text A folgt die Reihenfolge der Gaue einer Tradition, in der 14. u.äg. Gau zwischen den 16. und 17. u.äg. Gau geschoben wird. Vgl. etwa Helck, S. 133, O.
} 
Wie schon gesagt, schließt der Text der oberägyptischen Gaue mit einem Text, der den König als Horus legitimiert: „Komm auf mein Wort, mein Vater Osiris. Ich bin dein Sohn Horus, gerechtfertigt. Osiris Wenennefer, gerechtfertigt, großer Gott inmitten von Dendera, neige dein Angesicht gütig für den König von Ober- und Unterägypten, den Herrn der beiden Länder (leere Kartusche) ${ }^{\text {(195 }}$. Auf der unterägyptischen Seite wird der Anspruch der Gottheit auf den Empfang der Opfer und Ritualhandlungen dokumentiert: „Du bist der Lebende, durch dessen Anblick man lebt, alle Menschen leben von dir. Du bist der Herr des Windhauches, den er dem gibt, den er liebt, der abwendet alles (? twt-s ?) von dem, der deinen Namen nennt $(d m-r n-k)$. Ganz Ägypten führt für dich herbei seine Dinge. Du bist der Herr von ihnen allen. Du bist Osiris, machtvoll an seinem Leib, der entstanden ist aus (als ?) Amun-wer."

Es wurde schon darauf hingewiesen, daß Text B nicht einfach nur eine verkürzte Form des Textes A ist, auch nicht des ,geographischen“ Teiles davon. Dies läßt sich leicht deutlich machen, wenn man einmal die Passagen miteinander vergleicht. Zwar ist der fragende Anfang der Sätze meist gleich, aber bei den darauf folgenden Aussagen ergeben sich große Unterschiede. Bei den 20 Gauen Unterägyptens haben 15 Gaue einen unterschiedlichen Wortlaut. Bei den Gauen Oberägyptens sind die Unterschiede wesentlich geringer, aber insgesamt dürfte für Ober- und Unterägypten zusammen die Übereinstimmung nur bei etwa $60 \%$ liegen. In einigen Fällen gibt es bei unterschiedlichen Texten Übereinstimmungen in einzelnen Worten. So heißt es bei Sais im Text B: „Osiris, komm zu deinem Sohn“, bei Text A: „Osiris, komm zu den beiden Rechti“. Mendes hat bei Text B: „... so jauchzt deine Majestät über die Tätigkeit der Frauen“, Text A: „... so jauchzen die beiden Schwestern, wenn sie dich sehen ... “Man möchte annehmen, daß in diesen Fällen die Übereinstimmung der Worte nicht zufällig ist. Für viele Aussagen finden sich wörtliche oder sinngemäße Parallelen in ansonsten völlig anders gestalteten Texten zu den gleichen Gauen, wobei es etwa zu den Texten, die sich an Osiris wenden, Opet, Philae und RdM V, Taf. $3 \mathrm{ff}$., natürlich deutlichere Parallelen gibt als zu anderen. Aus den Übereinstimmungen muß man schließen, daß den ägyptischen Priestern, die mit der Formulierung solcher geographischen Texte befaßt waren, ein umfangreiches schriftliches und/oder mündliches Textmaterial zur Verfügung stand, aus dem sie einzelne Passagen wörtlich oder sinngemäß übernehmen konnten. Sie durften aber wohl im Rahmen der theologischen Vorgaben auch neue Gedanken in einen Text einbringen und sich dabei etwa der ägyptisch-wissenschaftlichen Methode bedienen, die Nomenklatur eines Gaues mit Hilfe der ägyptischen Variante der Etymologie, des Wortspiels, zu erklären und daraus Kenntnisse über seine Theologie zu erlangen. Wie und wann es zur Ausprägung neuer Texte kam, ist natürlich nicht nachzuweisen, da wir nicht über den Gesamtbestand ägyptischen Denkens verfügen. Man kann aber sicher sagen, daß die ägyptischen Priester, die für die Textredaktion verantwortlich waren, sich nicht mit der reinen Überlieferung einmal geprägter Texte begnügten, sondern stets um neue Zugänge zu theologischen Zusammenhängen bemüht waren.

\footnotetext{
${ }^{195}$ Dieses „sich dem König geneigt zeigen“ erweist sich durch das Akzeptieren der Opfer und Ritualhandlungen, die gleichzeitig oder nachfolgend durchgeführt werden.
} 


\section{ABK ÜRZUNGEN}

CD = E. Chassinat: Le temple de Dendara, Bd. I-IX, Le Caire $1934 \mathrm{ff}$. (Mitverfasser bzw. Verfasser ab Bd. VI: Fr. Daumas).

E. I = M. de Rochemonteix/E. Chassinat: Le temple d'Edfou I, MMAF 10, Paris 1897 (Verbesserte Neuauflage herausgegeben von S. Cau ville und D. Deva uchelle, Le Caire 1984ff.).

E. II-XIV = E. Chassinat: Le temple d'Edfou II-XIV, MMAF 11, MMAF 20-31, Paris/Le Caire 1918ff. K.O. = J. de Morgan: Kom Ombos, Vienne $1895 \mathrm{ff}$.

Mamm. D. = F. Daumas: Les mammisis de Dendara, Le Caire 1959.

Mamm. E. = E. Chassinat: Le mammisi d'Edfou, MIFAO 16, Le Caire 1939.

MD $\quad$ A. Mariette: Dendérah I-IV, Paris 1873.

Médamoud = E. Drioton: Rapport sur les fouilles de Médamoud (1925), FIFAO 3, Le Caire 1926.

Opet $\quad$ C C. de Wit: Les inscriptions du temple d'Opet à Karnak, BAe XI, Bruxelles $1958 \mathrm{ff}$.

Osirisreliquien = H. Beinlich: Die Osirisreliquien, ÄA 42, Wiesbaden 1984.

Philae $\quad$ G. Bénédite : Le temple de Philae, MMAF 13, Paris 1893.

RdM = H. Brugsch: Recueil des monuments égyptiens ..., I-VII, Leipzig $1862 \mathrm{ff} .($ Bd. III-VI $=$ J.

Tôd = J.-Cl. Grenier: Tôd, FIFAO 18, Le Caire 1980. 